\title{
Complete intersection toric ideals of oriented graphs and chorded-theta subgraphs
}

\author{
Isidoro Gitler · Enrique Reyes · Juan Antonio Vega
}

Received: 19 June 2012 / Accepted: 18 December 2012 / Published online: 4 May 2013

(C) Springer Science+Business Media New York 2013

\begin{abstract}
Let $G=(V, E)$ be a finite, simple graph. We consider for each oriented graph $G_{\mathcal{O}}$ associated to an orientation $\mathcal{O}$ of the edges of $G$, the toric ideal $P_{G_{\mathcal{O}}}$. In this paper we study those graphs with the property that $P_{G_{\mathcal{O}}}$ is a binomial complete intersection, for all $\mathcal{O}$. These graphs are called $\mathrm{CIO}$ graphs. We prove that these graphs can be constructed recursively as clique-sums of cycles and/or complete graphs. We introduce chorded-theta subgraphs and some of their properties. Also we establish that the $\mathrm{CIO}$ graphs are determined by the property that each chorded-theta has a transversal triangle. Finally we explicitly give the minimal forbidden induced subgraphs that characterize these graphs, these families of forbidden graphs are: prisms, pyramids, thetas and a particular family of wheels that we call $\theta$-partial wheels.
\end{abstract}

Keywords Toric ideals · Oriented graphs · Complete intersections · Forbidden induced subgraphs

\section{Introduction}

Let $G=(V, E)$ be a graph with $|E(G)|=q$ and whose vertex set is given by $V(G)=$ $\left\{x_{1}, \ldots, x_{n}\right\}$. An orientation $\mathcal{O}$ of the edges of $G$ is an assignment of a direction to each edge of $G$. Let $D=G_{\mathcal{O}}$ denote the oriented graph associated to an orientation $\mathcal{O}$ of the edges of $G$. In particular if $G$ is a complete graph, $G_{\mathcal{O}}$ is called a tournament.

I. Gitler (殴 · E. Reyes · J.A. Vega

Departamento de Matemáticas, Centro de Investigación y de Estudios Avanzados del IPN (CINVESTAV-IPN), Apartado Postal 14-740, 07000 México City, D.F., Mexico e-mail: igitler@math.cinvestav.edu.mx

E. Reyes

e-mail: ereyes@math.cinvestav.mx

J.A. Vega

e-mail: javega@ math.cinvestav.edu.mx 
To each oriented edge $e=\left(x_{i}, x_{j}\right)$ of $D$, we associate the vector $v_{e} \in\{0,1,-1\}^{n}$ defined as follows: the $i$ th entry is -1 , the $j$ th entry is 1 , and the remaining entries are zero. The incidence matrix $A_{D}$ of $D$ is the $n \times q$ matrix whose columns are the vectors of the form $v_{e}$, with $e$ an edge of $D$. The set of column vectors of $A_{D}$ will be denoted by $\mathcal{A}=\left\{v_{1}, \ldots, v_{q}\right\}$.

Consider the edge subring $k[D]:=k\left[x^{v_{1}}, \ldots, x^{v_{q}}\right] \subset k\left[x_{1}^{ \pm 1}, \ldots, x_{n}^{ \pm 1}\right]$ of $D$, where $v_{i}=\left(v_{i}^{1}, \ldots, v_{i}^{n}\right)$ and $x^{v_{i}}=x_{1}^{v_{i}^{1}} \ldots x_{n}^{v_{i}^{n}}$. Let $E(D)=\left\{t_{1}, \ldots, t_{q}\right\}$ be the edge set of $D$. There is an epimorphism of $k$-algebras given by

$$
\varphi: k\left[t_{1}, \ldots, t_{q}\right] \longrightarrow k[D], \quad \text { where } t_{i} \longmapsto x^{v_{i}},
$$

and $k\left[t_{1}, \ldots, t_{q}\right]$ is a polynomial ring. The kernel of $\varphi$, denoted by $P_{D}$, is called the toric ideal of $D$. This ideal has been thoroughly studied in $[5,6,12]$. The toric ideal $P_{D}$ is a prime ideal of height $q-n+r$, where $r$ is the number of connected components of $D . P_{D}$ is generated by binomials and $k[D]$ is a normal domain (see $[6,18]$ ). Thus any minimal generating set of $P_{D}$ must have at least $q-n+r$ elements, by the Principal Ideal Theorem (see [1]). If $P_{D}$ can be generated exactly by $q-n+r$ polynomials it is called a complete intersection. If these polynomials are binomials then $P_{D}$ is called a binomial complete intersection. In Sect. 2 we study the binomials of $P_{D}$. In particular, we prove that the primitive binomials of $P_{D}$ are the binomials associated to cycles. We also recover the result given in [6] that $P_{D}$ is generated by binomials associated to cycles without chords. If two graphs $G$ and $H$ each contain cliques of equal size, the clique-sum of $G$ and $H$ is formed from their disjoint union by identifying pairs of vertices in these two cliques to form a single shared clique.

It is known $[6,12]$ that any graph has at least one acyclic orientation such that the corresponding toric ideal is a binomial complete intersection. It is natural to ask what is the class of graphs with the property that $P_{G_{\mathcal{O}}}$ is generated by exactly $q-n+r$ binomials for every orientation $\mathcal{O}$ of $G$. For ease of exposition these graphs are called $\mathrm{CIO}$ graphs. In the bipartite case a graph $G$ is in this class if and only if $G$ is a ring graph (see [6]). Ring graphs are introduced in [5], they are those graphs for which their non trivial blocks (blocks that are not vertices or bridges) can be constructed as 2-clique-sums of cycles. In Sect. 3 we show that the $\mathrm{CIO}$ property is closed under induced subgraphs and we prove that $G$ is a $\mathrm{CIO}$ graph if and only if its blocks are $\mathrm{CIO}$ graphs.

In Sect. 4 we introduce the family of chorded-theta subgraphs and their transversal triangles. In this section we study the graphs $G$ that satisfy: for every chorded-theta of $G$ there exists a transversal triangle ( $\forall \theta \exists \Delta$-property). We call this type of graphs theta-ring graphs. In Theorem 4 we prove that these graphs can be constructed as clique-sums of cycles and complete graphs. Furthermore we show that they can be obtained as 0,1,2-clique-sums of chordal graphs and cycles. Theta-ring graphs are closed under induced subgraphs and we obtain the corresponding set of minimal forbidden induced subgraphs that characterize this family. These minimal forbidden induced subgraphs are: prisms, pyramids, thetas and $\theta$-partial wheels (partial wheels that are chorded-theta graphs).

Our main result in Sect. 5 is Theorem 6: $P_{G_{\mathcal{O}}}$ is a binomial complete intersection for every orientation $\mathcal{O}$ of $G$ if and only if $G$ is a theta-ring graph, i.e., the $\mathrm{CIO}$ 
property is equivalent to the $\forall \theta \exists \Delta$-property. As a consequence of this result, we see that $P_{G_{\mathcal{O}}}$ is a binomial complete intersection if $G_{\mathcal{O}}$ is a tournament. Using the results given in Sect. 4 and Sect. 5 we obtain the following equivalences:

$G$ is a
$\mathrm{CIO}$ graph $\Leftrightarrow \begin{gathered}G \text { is a theta-ring graph } \\ (G \text { has the } \forall \theta \exists \Delta \text {-property })\end{gathered} \Leftrightarrow \begin{gathered}G \text { is obtained by clique-sums } \\ \text { of complete graphs and cycles }\end{gathered}$

In particular, we recover that ring graphs are $\mathrm{CIO}$ graphs and that the converse also holds in the bipartite case.

The paper is essentially self contained. For unexplained terminology and notation on graph theory and toric ideals we refer to $[3,6,7,18]$. Some references for toric ideals associated to graphs (without orientation) are [5, 6, 8, 9, 13, 15-17].

\section{Toric ideals of oriented graphs}

Let $G$ be a graph and consider the oriented graph $D=G_{\mathcal{O}}$ associated to an orientation $\mathcal{O}$ of the edges of $G$. Let $\alpha=\left(\alpha_{1}, \ldots, \alpha_{q}\right) \in \mathbb{R}^{q}$, the support of $\alpha$ is the set $\operatorname{supp}(\alpha)=\left\{i \mid \alpha_{i} \neq 0\right\}$. Furthermore $\alpha=\alpha_{+}-\alpha_{-}$, where $\alpha_{+}$and $\alpha_{-}$are two nonnegative vectors with disjoint support. Recall that $\left\{v_{1}, \ldots, v_{q}\right\}$ is the set of column vectors of the incidence matrix $A_{D}$. We denote by $P_{D}$ the kernel of the epimorphism of $k$-algebras

$$
\varphi: k\left[t_{1}, \ldots, t_{q}\right] \longrightarrow k[D], \quad \text { where } t_{i} \longmapsto x^{v_{i}} .
$$

On the other hand we have the following linear map:

$$
\psi: \mathbb{Z}^{q} \longrightarrow \mathbb{Z}^{n}, \quad \text { where } e_{i} \longmapsto v_{i}
$$

i.e., $\psi$ is the linear map associated to the incidence matrix $A_{D}$ of $D$. If $0 \neq \alpha \in \mathbb{Z}^{q}$ we associate to it the binomial $t_{\alpha}=t^{\alpha_{+}}-t^{\alpha_{-}}$. Notice that $\alpha \in \operatorname{ker}(\psi)$ if and only if $t_{\alpha} \in P_{D}$. Given a cycle $C=\left(x_{j_{1}}, x_{j_{2}}, \ldots, x_{j_{k}}, x_{j_{k+1}}=x_{j_{1}}\right)$ of $D$, we split the edge set of $C$ in two disjoint sets $C_{+}$and $C_{-}$, where $C_{+}$is the set of clockwise oriented edges, i.e., $C_{+}=\left\{t_{s} \in E(C) \mid\right.$ there exist $i$ such that $\left.t_{s}=\left(x_{j_{i}}, x_{j_{i+1}}\right)\right\}$ and $C_{-}=C \backslash C_{+}$. Associated to $C$ we have the binomial

$$
t_{C}=\prod_{t_{i} \in C_{+}} t_{i}-\prod_{t_{i} \in C_{-}} t_{i} .
$$

This binomial belongs to $P_{D}$. If $C_{+}=\emptyset$ or $C_{-}=\emptyset$ we set $\prod_{t_{i} \in C_{+}} t_{i}=1$ or $\prod_{t_{i} \in C_{-}} t_{i}=1$.

In this section we study some properties of the binomials in $P_{D}$. In Theorem 1 we prove that if $b$ is a binomial in $P_{D}$, then there exists a binomial $b^{\prime}$ associated to a cycle whose monomials divide the monomials of $b$. Using this result we prove that the primitive binomials in $P_{D}$ are the ones associated with cycles and we recover the result in [6] that $P_{D}$ is generated by the binomials corresponding to cycles without chords. 
Lemma 1 Let $t^{\alpha}-t^{\beta}$ be a binomial in $P_{D}$ with $\operatorname{gcd}\left(t^{\alpha}, t^{\beta}\right)=1$. If $i \in \operatorname{supp}(\alpha)$ and $t_{i}=\left(x_{j_{1}}, x_{j_{2}}\right)$ then there exist edges $t_{i_{1}}$ and $t_{i_{2}}$ both different from $t_{i}$ such that $x_{j_{1}} \in V\left(t_{i_{1}}\right)$ and $x_{j_{2}} \in V\left(t_{i_{2}}\right)$. Furthermore if $t_{i_{2}}=\left(x_{j_{2}}, x_{j_{3}}\right)$, then $t_{i_{2}} \mid t^{\alpha}$ and if $t_{i_{2}}=$ $\left(x_{j_{3}}, x_{j_{2}}\right)$ then $t_{i_{2}} \mid t^{\beta}$.

Proof Since $t^{\alpha}-t^{\beta} \in P_{D}$ then

$$
\varphi\left(t_{1}^{\alpha_{1}}\right) \cdots \varphi\left(t_{q}^{\alpha_{q}}\right)=\varphi\left(t^{\alpha}\right)=\frac{m_{1}}{m_{2}}=\varphi\left(t^{\beta}\right)=\varphi\left(t_{1}^{\beta_{1}}\right) \cdots \varphi\left(t_{q}^{\beta_{q}}\right)
$$

where $\alpha=\left(\alpha_{1}, \ldots, \alpha_{q}\right)$ and $\beta=\left(\beta_{1}, \ldots, \beta_{q}\right)$. We can assume that $m_{1}$ and $m_{2}$ are monomials with $\operatorname{gcd}\left(m_{1}, m_{2}\right)=1$. Since $t_{i}=\left(x_{j_{1}}, x_{j_{2}}\right)$, we have $\varphi\left(t_{i}^{\alpha_{i}}\right)=\frac{x_{j_{2}}^{\alpha_{i}}}{x_{j_{1}}^{\alpha_{i}}}$ and $\alpha_{i} \neq 0$. If $x_{j_{2}} \mid m_{1}$ then there exists an edge $t_{i_{2}}$ such that $\beta_{i_{2}} \neq 0$ and $\varphi\left(t_{i_{2}}\right)=$ $\frac{x_{j_{2}}}{x_{j_{3}}}$ for some $x_{j_{3}}$. Thus $t_{i_{2}}=\left(x_{j_{3}}, x_{j_{2}}\right)$ and $t_{i_{2}} \mid t^{\beta}$. Furthermore $t_{i} \neq t_{i_{2}}$ because $\operatorname{gcd}\left(t^{\alpha}, t^{\beta}\right)=1$. Now, if $x_{j_{2}}$ does not divide $m_{1}$ then there exists an edge $t_{i_{2}}$ such that $\varphi\left(t_{i_{2}}\right)=\frac{x_{j_{4}}}{x_{j_{2}}}$ with $\alpha_{i_{2}} \neq 0$ and $i \neq i_{2}$. Hence, $t_{i_{2}}=\left(x_{j_{2}}, x_{j_{4}}\right)$ and $t_{i_{2}} \mid t^{\alpha}$, so $x_{j_{2}} \in V\left(t_{i_{2}}\right)$ and $t_{i_{2}} \neq t_{i}$.

In a similar way we prove that there exists an edge $t_{i_{1}}$ different from $t_{i}$ such that $x_{j_{1}} \in V\left(t_{i_{1}}\right)$.

Theorem 1 If $0 \neq t^{\alpha}-t^{\beta} \in P_{D}$ then there exists a cycle $C$ such that $t_{C}=t^{\alpha^{\prime}}-t^{\beta^{\prime}}$, $t^{\alpha^{\prime}} \mid t^{\alpha}$ and $t^{\beta^{\prime}} \mid t^{\beta}$.

Proof We can suppose that $\operatorname{gcd}\left(t^{\alpha}, t^{\beta}\right)=1$, otherwise we would have $\operatorname{gcd}\left(t^{\alpha}, t^{\beta}\right)=$ $m$. Thus, $t^{\alpha}=m t^{\gamma}, t^{\beta}=m t^{w}, \operatorname{gcd}\left(t^{\gamma}, t^{w}\right)=1$ and $t^{\gamma}-t^{w} \in P_{D}$. We set $\alpha=$ $\left(\alpha_{1}, \ldots, \alpha_{q}\right)$ and $\beta=\left(\beta_{1}, \ldots, \beta_{q}\right)$. Since $t^{\alpha}-t^{\beta} \neq 0$, then there exists $i$ such that $\alpha_{i} \neq 0$ or $\beta_{i} \neq 0$. We can suppose that $\alpha_{i} \neq 0$. By Lemma 1 if $x_{j_{1}} \in V\left(t_{i}\right)$ there exists an edge $t_{i_{1}}$ different from $t_{i}$ such that $x_{j_{1}} \in V\left(t_{i_{1}}\right)$ and $t_{i_{1}} \mid t^{\alpha} t^{\beta}$. Now if $V\left(t_{i_{1}}\right)=\left\{x_{j_{1}}, x_{j_{2}}\right\}$ then by Lemma 1 there exists an edge $t_{i_{2}}$ different from $t_{i_{1}}$ such that $x_{j_{2}} \in V\left(t_{i_{2}}\right)$ and $t_{i_{2}} \mid t^{\alpha} t^{\beta}$. We continue with this process and we obtain vertices $x_{j_{1}}, x_{j_{2}}, \ldots$ such that $\left\{x_{j_{k}}, x_{j_{k+1}}\right\} \in E(G)$. We take $l=\max \left\{i \mid x_{j_{i}} \neq x_{j_{k}}\right.$ for $\left.k<i\right\}$, then $x_{j_{l+1}}=x_{j_{s}}$ for some $s \leq l$. Therefore $C=\left(x_{j_{s}}, x_{j_{s+1}}, \ldots, x_{j_{l}}, x_{j_{l+1}}=x_{j_{s}}\right)$ is a cycle in $G$ and by construction if $t_{C}=t^{\alpha^{\prime}}-t^{\beta^{\prime}}$ then $t^{\alpha^{\prime}} t^{\beta^{\prime}} \mid t^{\alpha} t^{\beta}$. But, by Lemma 1 we have $t^{\alpha^{\prime}} \mid t^{\alpha}$ and $t^{\beta^{\prime}} \mid t^{\beta}$.

Definition 1 Let $C=\left(y_{1}, y_{2}, \ldots, y_{k}, y_{k+1}=y_{1}\right)$ be a cycle of $G$. $C$ is called an oriented cycle in $D=G_{\mathcal{O}}$ if $\left(y_{i}, y_{i+1}\right) \in E(D)$ for $i=1, \ldots, k$ (i.e. $C_{+}=\emptyset$ or $\left.C_{-}=\emptyset\right)$. Similarly a path $L=\left(y_{1}, \ldots, y_{s}\right)$ of $G$ is called an oriented path in $D$ if $\left(y_{i}, y_{i+1}\right) \in E(D)$ for $i=1, \ldots, s-1$. If $L$ is a path, we denote by $L^{o}$ the path $\left(y_{2}, \ldots, y_{s-1}\right)$, if $s=2$ then $L^{o}=\emptyset$.

Corollary 1 If $0 \neq 1-t^{\beta} \in P_{D}$ then there exists an oriented cycle $C$ in $D$.

Proof By Theorem 1, there is a cycle $C$ such that $t_{C}=t^{\alpha^{\prime}}-t^{\beta^{\prime}}, t^{\alpha^{\prime}} \mid 1$ and $t^{\beta^{\prime}} \mid t^{\beta}$. Then $t^{\alpha^{\prime}}=1$ and $C$ is an oriented cycle of $D$. 
Proposition 1 Let $f, g$ be binomials of $P_{D}$, such that $f=m_{1}-m_{2}$ and $g=$ $m_{1}-m_{2}^{\prime}$. If there is no oriented cycle in $D$ and $m_{2}^{\prime} \mid m_{2}$ then $f=g$.

Proof There exits a monomial $l$ such that $m_{2}=l m_{2}^{\prime}$ since $m_{2}^{\prime} \mid m_{2}$. On the other hand we have $0=\varphi(f)=\varphi\left(m_{1}\right)-\varphi(l) \varphi\left(m_{2}^{\prime}\right)$, then $\varphi\left(m_{1}\right)=\varphi(l) \varphi\left(m_{2}^{\prime}\right)$. But $\varphi\left(m_{1}\right)=$ $\varphi\left(m_{2}^{\prime}\right)$ because $g \in P_{D}$. Hence, $\varphi(1)=\varphi(l)$ and $(1-l) \in P_{D}$. Using Corollary 1 and since there is no oriented cycle in $D$ we have $l=1$ and $f=g$.

Corollary 2 If $0 \neq t^{\alpha}-t^{\beta}=f$ in $P_{D}$ with $\operatorname{gcd}\left(t^{\alpha}, t^{\beta}\right)=1$, then there exist cycles $C_{1}, \ldots, C_{s}$ such that $t^{\alpha}=t^{\alpha_{1}} \cdots t^{\alpha_{s}}$ and $t^{\beta}=t^{\beta_{1}} \cdots t^{\beta_{s}}$ where $t_{C_{i}}=t^{\alpha_{i}}-t^{\beta_{i}}$ for $i=1, \ldots, s$.

Proof By Theorem 1 there exists a cycle $C_{1}$ with $t_{C_{1}}=t^{\alpha_{1}}-t^{\beta_{1}}$ such that $t^{\alpha}=t^{\alpha_{1}} t^{\alpha^{\prime}}$ and $t^{\beta}=t^{\beta_{1}} t^{\beta^{\prime}}$. Since $f=t^{\alpha}-t^{\beta}$ in $P_{D}$ then $\varphi(f)=0$ and

$$
\varphi\left(t^{\alpha_{1}}\right) \varphi\left(t^{\alpha^{\prime}}\right)=\varphi\left(t^{\alpha}\right)=\varphi\left(t^{\beta}\right)=\varphi\left(t^{\beta_{1}}\right) \varphi\left(t^{\beta^{\prime}}\right) .
$$

But $t_{C_{1}} \in P_{D}$ then $\varphi\left(t^{\alpha_{1}}\right)=\varphi\left(t^{\beta_{1}}\right)$. Hence $\varphi\left(t^{\alpha^{\prime}}\right)=\varphi\left(t^{\beta^{\prime}}\right)$ and $f_{1}=t^{\alpha^{\prime}}-t^{\beta^{\prime}} \in P_{D}$. Since $\operatorname{gcd}\left(t^{\alpha^{\prime}}, t^{\beta^{\prime}}\right)=1$, if $f_{1}=0$ then $t^{\alpha^{\prime}}=t^{\beta^{\prime}}=1$ and $f=t_{C_{1}}$. Now, if $f_{1} \neq 0$ by Theorem 1 there exists a cycle $C_{2}$ with $t_{C_{2}}=t^{\alpha_{2}}-t^{\beta_{2}}$ such that $t^{\alpha^{\prime}}=t^{\alpha_{2}} t^{\alpha^{\prime \prime}}$ and $t^{\beta^{\prime}}=t^{\beta_{2}} t^{\beta^{\prime \prime}}$. Furthermore $t^{\alpha}=t^{\alpha_{1}} t^{\alpha_{2}} t^{\alpha^{\prime \prime}}, t^{\beta}=t^{\beta_{1}} t^{\beta_{2}} t^{\beta^{\prime \prime}}$ and $f_{2}=t^{\alpha^{\prime \prime}}-t^{\beta^{\prime \prime}} \in P_{D}$. We continue this process to obtain the result.

Definition 2 A binomial $t^{\alpha}-t^{\beta}$ in $P_{D}$ is called primitive if there exists no other binomial $t^{\alpha^{\prime}}-t^{\beta^{\prime}} \in P_{D}$ such that $t^{\alpha^{\prime}}$ divides $t^{\alpha}$ and $t^{\beta^{\prime}}$ divides $t^{\beta}$.

Proposition 2 The primitive binomials of $P_{D}$ are the binomials of $P_{D}$ associated to cycles.

Proof Let $g=t^{\alpha}-t^{\beta}$ be a primitive binomial. By Theorem 1 there exists a cycle $C$ such that $t_{C}=t^{\alpha^{\prime}}-t^{\beta^{\prime}}, t^{\alpha^{\prime}} \mid t^{\alpha}$ and $t^{\beta^{\prime}} \mid t^{\beta}$. But $g$ is primitive then $g=t_{C}$, i.e., $g$ is associated to $C$. Therefore to obtain the result it is only necessary to prove that the binomials associated to cycles are primitive. So, let $t_{C}=t^{\gamma}-t^{w}$ be the binomial associated to $C$, then $\operatorname{gcd}\left(t^{\gamma}, t^{w}\right)=1$. Suppose that there exists a binomial $f=t^{\gamma^{\prime}}-$ $t^{w^{\prime}} \in P_{D}$ such that $t^{\gamma^{\prime}} \mid t^{\gamma}$ and $t^{w^{\prime}} \mid t^{w}$. Then, by Theorem 1 there exists a cycle $C^{\prime}$ such that $t_{C^{\prime}}=t^{\gamma^{\prime \prime}}-t^{w^{\prime \prime}}, t^{\gamma^{\prime \prime}} \mid t^{\gamma^{\prime}}$ and $t^{w^{\prime \prime}} \mid t^{w^{\prime}}$. Hence, $t^{\gamma^{\prime \prime}} \mid t^{\gamma}$ and $t^{w^{\prime \prime}} \mid t^{w}$, this implies $E\left(C^{\prime}\right) \subset E(C)$. Thus, $C^{\prime}=C, t_{C^{\prime}}=t_{C}$ and $t_{C}=f$. Therefore $t_{C}$ is primitive.

Proposition 3 The toric ideal $P_{D}$ is generated by the binomials associated to cycles: $P_{D}=\left(t_{C} \mid C\right.$ is cycle of $\left.D\right)$.

Proof First we observe that

$$
t^{\alpha_{1}} t^{\alpha_{2}}-t^{\beta_{1}} t^{\beta_{2}}=t^{\alpha_{1}}\left(t^{\alpha_{2}}-t^{\beta_{2}}\right)+t^{\beta_{2}}\left(t^{\alpha_{1}}-t^{\beta_{1}}\right)
$$


then $t^{\alpha_{1}} t^{\alpha_{2}}-t^{\beta_{1}} t^{\beta_{2}} \in\left(t^{\alpha_{1}}-t^{\beta_{1}}, t^{\alpha_{2}}-t^{\beta_{2}}\right)$. Now, if $g=t^{\alpha}-t^{\beta} \in P_{D}$ then by Corollary 2, there exist cycles $C_{1}, \ldots, C_{s}$ in $D$ with $t_{C_{i}}=t^{\alpha_{i}}-t^{\beta_{i}}$ such that $t^{\alpha}=m t^{\alpha^{\prime}}=m t^{\alpha_{1}} \cdots t^{\alpha_{s}}$ and $t^{\beta}=m t^{\beta^{\prime}}=m t^{\beta_{1}} \cdots t^{\beta_{s}}$ where $m=\operatorname{gcd}\left(t^{\alpha}, t^{\beta}\right)$. By the first observation we have $g=m\left(t^{\alpha^{\prime}}-t^{\beta^{\prime}}\right) \in\left(t_{C_{1}}, \ldots, t_{C_{s}}\right)$. Therefore $P_{D}=\left(t_{C} \mid\right.$ $C$ is cycle of $D$ ).

The last result also follows from the fact that the Graver basis is a generating set for the ideal.

Lemma 2 Let $C_{1}, C_{2}$ be two cycles of $D$ whose intersection is an oriented path $P$. Then $C_{3}=\left(C_{1} \cup C_{2}\right) \backslash P^{o}$ is a cycle and $t_{C_{3}} \in\left(t_{C_{1}}, t_{C_{2}}\right)$.

Proof We can assume that $C_{1}=\left(x=x_{0}, x_{1}, \ldots, x_{r_{1}}=y, y_{1}, \ldots, y_{r_{2}}, x\right), C_{2}=$ $\left(x=x_{0}, x_{1}, \ldots, x_{r_{1}}=y, z_{1}, \ldots, z_{r_{3}}, x\right)$ and $C_{1} \cap C_{2}=P=\left(x, x_{1}, \ldots, x_{r_{1}-1}, y\right)$ where $\left(x_{i}, x_{i+1}\right) \in E(D)$ for $i=0, \ldots, r_{1}-1$, then $C_{3}=\left(C_{1} \cup C_{2}\right) \backslash P^{o}=$ $\left(y, y_{1}, \ldots, y_{r_{2}}, x, z_{r_{3}}, \ldots, z_{1}, y\right)$. Thus, $C_{3}$ is a cycle. Without loss of generality we can suppose that $t_{i+1}=\left(x_{i}, x_{i+1}\right)$ for $i=0, \ldots, r_{1}-1$. Hence, $t_{C_{1}}=t^{\alpha} t^{\alpha_{1}}-t^{\beta_{1}}$ and $t_{C_{2}}=t^{\alpha} t^{\alpha_{2}}-t^{\beta_{2}}$ where $\alpha=\sum_{i=1}^{r_{1}} e_{i}$. Furthermore $t_{C_{3}}=t^{\alpha_{1}} t^{\beta_{2}}-t^{\alpha_{2}} t^{\beta_{1}}$. But $t^{\alpha_{1}} t^{\beta_{2}}-t^{\alpha_{2}} t^{\beta_{1}}=t^{\alpha_{2}} t_{C_{1}}-t^{\alpha_{1}} t_{C_{2}}$ therefore, $t_{C_{3}} \in\left(t_{C_{1}}, t_{C_{2}}\right)$.

Proposition 4 ([6]) $P_{D}$ is generated by the set of binomials corresponding to cycles without chords.

Proof By Proposition 3 it is only necessary to prove that if

$$
C=\left(x_{1}, x_{2}, \ldots, x_{s}, x_{s+1}=x_{1}\right)
$$

is a cycle of $G$ and $e=\left\{x_{i}, x_{j}\right\}$ is a chord of $C$, then $t_{C} \in\left(t_{C_{1}}, t_{C_{2}}\right)$ where $C_{1}=$ $\left(x_{i}, x_{i+1}, \ldots, x_{j}, x_{i}\right)$ and $C_{2}=\left(x_{1}, \ldots, x_{i}, x_{j}, x_{j+1}, \ldots, x_{s}, x_{1}\right)$. But the intersection of $C_{1}$ and $C_{2}$ is the edge $e$, then we obtain the result by Lemma 2 .

\section{$3 \mathrm{CIO}$ graphs and toric ideals}

Let $G$ be a graph, with $q$ edges and $r$ connected components such that $V(G)=$ $\left\{x_{1}, \ldots, x_{n}\right\}$. For ease of exposition we will say that $G$ is $\mathrm{CIO}$ if the toric ideal $P_{G_{\mathcal{O}}}$ is a binomial complete intersection for each orientation $\mathcal{O}$. Recall that this means that $P_{G_{\mathcal{O}}}$ is generated by $q-n+r$ binomials, for every orientation $\mathcal{O}$ of $G$. The main technical result in this section is Theorem 2, using this theorem and Lemma 6 we prove that the $\mathrm{CIO}$ property is closed under induced subgraphs. Also we prove that a graph is $\mathrm{CIO}$ if and only if its blocks are $\mathrm{CIO}$.

Recall that if $v \in V(G)$, the neighborhood of $v$ is the set $N_{G}(v)=\{w \in V(G) \mid$ $\{v, w\} \in E(G)\}$. We take $x \in V(G), G^{\prime}=G \backslash x$ and $\mathcal{O}^{\prime}$ an orientation of $G^{\prime}$. If $N_{G}(x)=\left\{x_{1}, \ldots, x_{s}\right\}$, then we define the orientation $\mathcal{O}_{x}$ of $G$ as follows: set $E\left(G_{\mathcal{O}_{x}}\right)=E\left(G_{\mathcal{O}^{\prime}}^{\prime}\right) \cup\left\{\left(x, x_{1}\right), \ldots,\left(x, x_{s}\right)\right\}$. In the following three lemmas assume that $E\left(G_{\mathcal{O}_{x}}\right)=\left\{t_{1}, \ldots, t_{q}\right\}$, where $t_{i}=\left(x, x_{i}\right)$ for $i=1, \ldots, s$. We denote the oriented graph $G_{\mathcal{O}_{x}}$ by $D_{x}$ (i.e. $D_{x}=G_{\mathcal{O}_{x}}$ ). 
Lemma 3 Let $t^{\alpha}-t^{\beta}$ be a binomial in $P_{D_{x}}$. If $\alpha=\left(\alpha_{1}, \ldots, \alpha_{q}\right)$ and $\beta=$ $\left(\beta_{1}, \ldots, \beta_{q}\right)$, then $\alpha \cdot(\underbrace{1, \ldots, 1}_{s}, 0, \ldots, 0)=\beta \cdot(\underbrace{1, \ldots, 1}_{s}, 0, \ldots, 0)$, i.e., $\sum_{i=1}^{s} \alpha_{i}=$ $\sum_{i=1}^{s} \beta_{i}$.

Proof We have $t_{i}=\left(x, x_{i}\right)$ for $i=1, \ldots, s$ thus, $\varphi\left(t_{i}\right)=x_{i} x^{-1}$. Hence, $\varphi\left(t^{\alpha}\right)=$ $\frac{m_{1}}{x^{k_{1} m_{2}}}$ and $\varphi\left(t^{\beta}\right)=\frac{n_{1}}{x^{k_{2} n_{2}}}$, where $m_{1}, m_{2}, n_{1}$ and $n_{2}$ are monomials. Also set $\operatorname{gcd}\left(x, m_{1}\right)=\operatorname{gcd}\left(x, m_{2}\right)=\operatorname{gcd}\left(x, n_{1}\right)=\operatorname{gcd}\left(x, n_{2}\right)=1$. Furthermore $k_{1}=\sum_{i=1}^{s} \alpha_{i}$ and $k_{2}=\sum_{i=1}^{s} \beta_{i}$. On the other hand $t^{\alpha}-t^{\beta} \in P_{D_{x}}$ then $\varphi\left(t^{\beta}\right)=\varphi\left(t^{\alpha}\right)$. Therefore, $\frac{m_{1}}{x^{k_{1} m_{2}}}=\frac{n_{1}}{x^{k_{2} n_{2}}}$ and $k_{1}=\sum_{i=1}^{s} \alpha_{i}=\sum_{i=1}^{s} \beta_{i}=k_{2}$.

Corollary 3 Let $t^{\alpha}-t^{\beta}$ be a binomial in $P_{D_{x}}$. If there exists $a \in\{1, \ldots, s\}$ such that $t_{a} \mid t^{\alpha}$ then there exists $b \in\{1, \ldots, s\}$ such that $t_{b} \mid t^{\beta}$.

Lemma 4 Let $f=t^{\alpha}-t^{\beta}$ be a binomial in $P_{D_{x}} \cap k\left[t_{s+1}, \ldots, t_{q}\right]$. If $B$ is a generating set of binomials of $P_{D_{x}}$ and $B^{\prime}=B \cap k\left[t_{s+1}, \ldots, t_{q}\right]$ then $f \in\left(B^{\prime}\right) \subset k\left[t_{s+1}, \ldots, t_{q}\right]$.

Proof Set $B=\left\{g_{\lambda}\right\}_{\lambda \in \Omega}$. Since $P_{D_{x}}=(B)$, then $f=t^{\alpha}-t^{\beta}=\sum_{\lambda \in \Omega} f_{\lambda} g_{\lambda}$, where $f_{\lambda} \in k\left[t_{1}, \ldots, t_{q}\right]$. We set in the equation above $t_{1}=t_{2}=\cdots=t_{s}=0$. By Corollary 3

$$
\left.g_{\lambda}\right|_{t_{1}=t_{2}=\cdots=t_{s}=0}= \begin{cases}g_{\lambda} & \text { if } g_{\lambda} \in B^{\prime} \\ 0 & \text { if } g_{\lambda} \notin B^{\prime}\end{cases}
$$

Hence, we obtain $f=\left.f\right|_{t_{1}=t_{2}=\cdots=t_{s}=0}=\sum_{g_{\lambda} \in B^{\prime}} f_{\lambda}^{\prime} g_{\lambda}$, where $f_{\lambda}^{\prime}=$ $\left.f_{\lambda}\right|_{t_{1}=t_{2}=\cdots=t_{s}=0} \in k\left[t_{s+1}, \ldots, t_{q}\right]$. Therefore $f \in\left(B^{\prime}\right)$.

Lemma 5 If $G^{\prime}=G \backslash x$ is a connected graph then for every $j_{1}, j_{2} \in\{1, \ldots, s\}$ there exists a binomial $t_{j_{1}} m_{j_{1}}-t_{j_{2}} m_{j_{2}}$ in $P_{D_{x}}$ with $m_{j_{1}}, m_{j_{2}} \in k\left[t_{s+1}, \ldots, t_{q}\right]$.

Proof We know that $t_{j_{1}}=\left(x, x_{j_{1}}\right)$ and $t_{j_{2}}=\left(x, x_{j_{2}}\right)$. Since $G^{\prime}$ is connected, there exists a path $\mathcal{L}_{j_{1}, j_{2}}$ in $D^{\prime}=G_{\mathcal{O}^{\prime}}^{\prime}$ between $x_{j_{1}}$ and $x_{j_{2}}$. Thus, $C_{j_{1}, j_{2}}=$ $\left(x, x_{j_{1}}, \mathcal{L}_{j_{1}, j_{2}}, x_{j_{2}}, x\right)$ is a cycle in $D_{x}$ and $t_{C_{j_{1}, j_{2}}}=t_{j_{1}} m-t_{j_{2}} m^{\prime}$ with $m, m^{\prime} \in$ $k\left[t_{s+1}, \ldots, t_{q}\right]$. Therefore $t_{C_{j_{1}, j_{2}}} \in P_{D_{x}}$.

Theorem 2 Let $G$ be a connected graph. If there exists a vertex $x$ which is not a cut vertex of $G$ such that $G^{\prime}=G \backslash x$ is not a $C I \mathcal{O}$ graph then $G$ is not a $C I \mathcal{O}$ graph.

Proof Set $|V(G)|=n,|E(G)|=q$ and $\left|E(G) \backslash E\left(G^{\prime}\right)\right|=s$. Since $G^{\prime}$ is not a CIO graph there exists an oriented graph $D^{\prime}=G_{\mathcal{O}^{\prime}}^{\prime}$ associated to an orientation $\mathcal{O}^{\prime}$ of $G^{\prime}$ such that $P_{D^{\prime}}$ is not generated by ht $\left(P_{D^{\prime}}\right)$ binomials. We take the orientation $\mathcal{O}_{x}$ of $G$ given by $E\left(G_{\mathcal{O}_{x}}\right)=E\left(G_{\mathcal{O}^{\prime}}^{\prime}\right) \cup\left\{\left(x, x_{1}\right), \ldots,\left(x, x_{s}\right)\right\}$, where $N_{G}(x)=\left\{x_{1}, \ldots, x_{s}\right\}$ and $D_{x}=G_{\mathcal{O}_{x}}$. We can suppose that $t_{i}=\left(x, x_{i}\right)$ for $1 \leq i \leq s$, i.e., $E\left(D_{x}\right) \backslash E\left(D^{\prime}\right)=$ $\left\{t_{1}, \ldots, t_{s}\right\}$. We take $\mathcal{G}=\left\{f_{1}, \ldots, f_{k}\right\}$ a minimum generating set of binomials of $P_{D_{x}}$ and we define $\mathcal{G}^{\prime}=\mathcal{G} \cap k\left[t_{s+1}, \ldots, t_{q}\right]$. By Lemma $4, P_{D^{\prime}}=\left(g \mid g \in \mathcal{G}^{\prime}\right)$ and 
$\operatorname{ht}\left(P_{D^{\prime}}\right)=(q-s)-(n-1)+1$. Hence $\left|\mathcal{G}^{\prime}\right|>(q-s)-(n-1)+1$. Now, we define the auxiliary graph $\mathcal{H}$ with $V(\mathcal{H})=\left\{t_{1}, \ldots, t_{s}\right\}$ and

$$
E(\mathcal{H})=\left\{\begin{array}{l|l}
\left\{t_{i}, t_{j}\right\} & \begin{array}{l}
\text { there exist monomials } m_{i}, m_{j} \in k\left[t_{s+1}, \ldots, t_{q}\right] \text { such that } \\
t_{i} m_{i}-t_{j} m_{j} \in \mathcal{G} \text { or } t_{j} m_{j}-t_{i} m_{i} \in \mathcal{G} \text { with } i, j \in\{1, \ldots, s\}
\end{array}
\end{array}\right\} .
$$

If $A=\left\{t_{i} m_{i}-t_{j} m_{j} \in \mathcal{G} \mid\left\{t_{i}, t_{j}\right\} \in E(\mathcal{H})\right\}$ then $|A|=|E(\mathcal{H})|$. Also $A \cup \mathcal{G}^{\prime} \subset \mathcal{G}$ and $A \cap \mathcal{G}^{\prime}=\emptyset$. Hence we have $|E(\mathcal{H})|+\left|\mathcal{G}^{\prime}\right| \leq|\mathcal{G}|$. Now, if $\mathcal{H}$ is connected, then $|E(\mathcal{H})| \geq|V(\mathcal{H})|-1=s-1$. Furthermore, since $\left|\mathcal{G}^{\prime}\right|>(q-s)-(n-1)+1$, then $|\mathcal{G}|>(s-1)+(q-s)-(n-1)+1=q-n+1=h t\left(P_{D_{x}}\right)$. Thus, $P_{D_{x}}$ is not generated by $q-n+1$ binomials.

Thus, to obtain the result it is only necessary to prove that $\mathcal{H}$ is connected. Suppose, by way of contradiction, that the connected components of $\mathcal{H}$ are $\mathcal{H}_{1}, \ldots, \mathcal{H}_{p}$ with $p>1$. By Lemma 5 there exists a binomial $t_{1} m_{1}-t_{j} m_{j} \in P_{D_{x}}$ such that $t_{1} \in V\left(\mathcal{H}_{1}\right)$ and $t_{j} \in V\left(\mathcal{H}_{2}\right)$, where $m_{1}, m_{j}$ are monomials in $k\left[t_{s+1}, \ldots, t_{q}\right]$ and $j \in\{2, \ldots, s\}$. Recall that each $f_{i} \in \mathcal{G}$ has the form $f_{i}=t^{\alpha_{i}}-t^{\beta_{i}}$, where $t^{\alpha_{i}}, t^{\beta_{i}}$ are monomials in $k\left[t_{1}, \ldots, t_{q}\right]$, then

$$
t_{1} m_{1}-t_{j} m_{j}=\sum_{i=1}^{k} g_{i}\left(t^{\alpha_{i}}-t^{\beta_{i}}\right), \quad \text { with } g_{i} \in k\left[t_{1}, \ldots, t_{q}\right]
$$

If $\alpha_{i}=\left(\alpha_{1}^{i}, \ldots, \alpha_{q}^{i}\right)$ and $\beta_{i}=\left(\beta_{1}^{i}, \ldots, \beta_{q}^{i}\right)$, we take $\alpha_{i}^{\prime}=\left(\alpha_{1}^{i}, \ldots, \alpha_{s}^{i}, 0, \ldots, 0\right)$ and $\beta_{i}^{\prime}=\left(\beta_{1}^{i}, \ldots, \beta_{s}^{i}, 0, \ldots, 0\right)$; observe by Lemma $3, \operatorname{deg}\left(t^{\alpha_{i}^{\prime}}\right)=\operatorname{deg}\left(t^{\beta_{i}^{\prime}}\right)$ for $i \in\{1, \ldots, k\}$. Moreover, $\alpha_{i}^{\prime}=0 \Leftrightarrow \beta_{i}^{\prime}=0$, and in this case, we have $\left(t^{\alpha_{i}^{\prime}}-\right.$ $\left.t^{\beta_{i}^{\prime}}\right)\left.\right|_{t_{s+1}=\cdots=t_{q}=1}=0$. Hence, if we set in the equation above $t_{s+1}=\cdots=t_{q}=1$, we obtain

$$
t_{1}-t_{j}=\sum_{\operatorname{deg}\left(t^{\alpha_{i}^{\prime}}\right)=1} g_{i}^{\prime}\left(t^{\alpha_{i}^{\prime}}-t^{\beta_{i}^{\prime}}\right)+\sum_{\operatorname{deg}\left(t^{\alpha_{i}^{\prime}}\right)>1} g_{i}^{\prime}\left(t^{\alpha_{i}^{\prime}}-t^{\beta_{i}^{\prime}}\right),
$$

where $g_{i}^{\prime}=\left.g_{i}\right|_{t_{s+1}=\cdots=t_{q}=1}$. Furthermore, we can write $g_{i}^{\prime}=a_{i}+\lambda_{i}$ with $a_{i} \in$ $k\left[t_{1}, \ldots, t_{s}\right]$ and $\lambda_{i} \in k$, such that if $a_{i} \neq 0$ then $\operatorname{deg}\left(a_{i}\right)>0$. Thus,

$$
t_{1}-t_{j}=\sum_{\operatorname{deg}\left(t^{\alpha_{i}^{\prime}}\right)=1} \lambda_{i}\left(t^{\alpha_{i}^{\prime}}-t^{\beta_{i}^{\prime}}\right)+g^{\prime}
$$

where $g^{\prime}=\sum_{\operatorname{deg}\left(t^{\alpha_{i}^{\prime}}\right)=1} a_{i}\left(t^{\alpha_{i}^{\prime}}-t^{\beta_{i}^{\prime}}\right)+\sum_{\operatorname{deg}\left(t^{\alpha_{i}}\right)>1} g_{i}^{\prime}\left(t^{\alpha_{i}^{\prime}}-t^{\beta_{i}^{\prime}}\right)$. Hence we have either $\operatorname{deg}\left(g^{\prime}\right)>1$ or $g^{\prime}=0$. If we suppose $\operatorname{deg}\left(g^{\prime}\right)>1$, then

$$
t_{1}-t_{j}-\sum_{\operatorname{deg}\left(t^{\alpha_{i}^{\prime}}\right)=1} \lambda_{i}\left(t^{\alpha_{i}^{\prime}}-t^{\beta_{i}^{\prime}}\right)=g^{\prime}
$$


however, the left side has degree at most one, but $\operatorname{deg}\left(g^{\prime}\right)>1$, which is a contradiction. Therefore $g^{\prime}=0$, so

$$
\begin{aligned}
t_{1}-t_{j} & =\sum_{\operatorname{deg}\left(t^{\alpha_{i}^{\prime}}\right)=1} \lambda_{i}\left(t^{\alpha_{i}^{\prime}}-t^{\beta_{i}^{\prime}}\right) \\
& =\sum_{\left\{t_{i_{1}}, t_{i_{2}}\right\} \in E\left(\mathcal{H}_{1}\right)} \lambda_{i_{1}, i_{2}}^{1}\left(t_{i_{1}}-t_{i_{2}}\right)+\cdots+\sum_{\left\{t_{i_{1}}, t_{i_{2}}\right\} \in E\left(\mathcal{H}_{p}\right)} \lambda_{i_{1}, i_{2}}^{p}\left(t_{i_{1}}-t_{i_{2}}\right),
\end{aligned}
$$

where $\lambda_{i}=\lambda_{i_{1}, i_{2}}^{u}$ if $t^{\alpha_{i}^{\prime}}=t_{i_{1}}, t^{\beta_{i}^{\prime}}=t_{i_{2}}$ and $\left\{t_{i_{1}}, t_{i_{2}}\right\} \in E\left(\mathcal{H}_{u}\right)$. In the last equation we set $t_{\ell}=1$ if $t_{\ell} \in V\left(\mathcal{H}_{1}\right)$ and $t_{\ell}=0$ otherwise, and we obtain

$$
\begin{aligned}
1-0= & \sum_{\left\{t_{i_{1}}, t_{i_{2}}\right\} \in E\left(\mathcal{H}_{1}\right)} \lambda_{i_{1}, i_{2}}^{1}(1-1)+\sum_{\left\{t_{i_{1}}, t_{i_{2}}\right\} \in E\left(\mathcal{H}_{2}\right)} \lambda_{i_{1}, i_{2}}^{2}(0-0)+\cdots \\
& +\sum_{\left\{t_{i_{1}}, t_{i_{2}}\right\} \in E\left(\mathcal{H}_{p}\right)} \lambda_{i_{1}, i_{2}}^{p}(0-0)=0
\end{aligned}
$$

since $t_{1} \in V\left(\mathcal{H}_{1}\right)$ and $t_{j} \in V\left(\mathcal{H}_{2}\right)$. This is a contradiction. Therefore $\mathcal{H}$ is connected.

Definition 3 Let $G$ be a graph. A subgraph $H$ of $G$ is a called an induced subgraph of $G$ if $E(H)=\{e \in E(G) \mid e \subseteq V(H)\}$. Furthermore if $X \subseteq V(G)$ then we denote by $[X]_{G}$ the induced subgraph $H$ of $G$ such that $V(H)=X$, and in this case we say that $H$ is induced by $X$.

Lemma 6 Let $G$ be a connected graph. If $G^{\prime}$ is a proper induced connected subgraph of $G$, then there exists $x \in V(G) \backslash V\left(G^{\prime}\right)$ such that $G \backslash x$ is connected.

Proof Set $|V(G)|=n$. If $v \in V(G) \backslash V\left(G^{\prime}\right)$, there exists $G_{v}$, a connected component of $G \backslash v$ such that $G^{\prime} \subset G_{v}$. We define $r_{v}=\left|V\left(G_{v}\right)\right|$. Let $x$ be the vertex in $V(G) \backslash V\left(G^{\prime}\right)$ with $r_{x}$ maximal. Let $G_{1}, \ldots, G_{k}$ be the connected components of $G \backslash x$ with $G^{\prime} \subset G_{1}$, i.e., $G_{v}=G_{1}$. Hence, $\left|V\left(G_{1}\right)\right|=r_{x}$. Suppose that $r_{x}<n-1$, then $k>1$. Thus, there is $y \in V\left(G_{2}\right) \subset V(G) \backslash V\left(G^{\prime}\right)$. Since $G$ is connected, we have $x$ is adjacent to a vertex of $G_{1}$. Hence $\left[V\left(G_{1}\right) \cup x\right]_{G}$ is contained in a connected component of $G \backslash y$. Then $r_{y}>r_{x}$, this is a contradiction. Therefore $r_{x}=n-1, k=1$ and $G \backslash x$ is connected.

Lemma 7 Let $G_{1}, G_{2}$ be $C I \mathcal{O}$ subgraphs of $G$, such that $G=G_{1} \cup G_{2}$. If $\mid V\left(G_{1}\right) \cap$ $V\left(G_{2}\right) \mid \leq 1$ then $G$ is a $C I \mathcal{O}$ graph.

Proof Let $D=G_{\mathcal{O}}$ be the oriented graph associated to an orientation $\mathcal{O}$ of $G$ and $\mathcal{O}_{i}$ the orientation of $G_{i}$ induced by $\mathcal{O}$. We set $D_{i}=\left(G_{i}\right)_{\mathcal{O}_{i}}$. Then for $i=1,2$ there exists a binomial generating set $\mathcal{G}_{i}$ of $P_{D_{i}}$ such that $\left|\mathcal{G}_{i}\right|=q_{i}-n_{i}+r_{i}$ where $q_{i}=$ $\left|E\left(G_{i}\right)\right|, n_{i}=\left|V\left(G_{i}\right)\right|$ and $r_{i}$ is the number of the connected components of $G_{i}$. Since $\left|V\left(D_{1}\right) \cap V\left(D_{2}\right)\right| \leq 1$, we see that if $C$ is a cycle of $D$ then either $C \subset D_{1}$ or 
$C \subset D_{2}$. Hence, $t_{C} \in\left(\mathcal{G}_{1} \cup \mathcal{G}_{2}\right)$. So $\mathcal{G}=\mathcal{G}_{1} \cup \mathcal{G}_{2}$ is a binomial generating set of $P_{D}$. Since $E\left(G_{1}\right) \cap E\left(G_{2}\right)=\emptyset$ then $\mathcal{G}_{1} \cap \mathcal{G}_{2}=\emptyset$ and

$$
|\mathcal{G}|=\left|\mathcal{G}_{1}\right|+\left|\mathcal{G}_{2}\right|=\left(q_{1}+q_{2}\right)-\left(n_{1}+n_{2}\right)+\left(r_{1}+r_{2}\right) .
$$

On the other hand $|E(G)|=q_{1}+q_{2}$ and the cardinality of $V(G)$ is

$$
|V(G)|= \begin{cases}n_{1}+n_{2} & \text { if } V\left(D_{1}\right) \cap V\left(D_{2}\right)=\emptyset \\ n_{1}+n_{2}-1 & \text { if }\left|V\left(D_{1}\right) \cap V\left(D_{2}\right)\right|=1\end{cases}
$$

but if $r$ is the number of components of $G$ then $r=r_{1}+r_{2}$ in the first case and $r=r_{1}+r_{2}-1$ in the second case. Therefore $|\mathcal{G}|=|E(G)|-|V(G)|+r=\operatorname{ht}\left(P_{D}\right)$ and $G$ is a $\mathrm{CI} \mathcal{O}$ graph.

Proposition 5 Let $G$ be a graph. $G$ is a CIO graph if and only if its connected components are $\mathrm{CIO}$ graphs.

Proof $(\Rightarrow)$ By induction on $|V(G)|$. Let $G_{1}, \ldots, G_{k}$ be the connected components of $G$. We can suppose that $k>1$. By Lemma 6 there exists $x_{i} \in V\left(G_{i}\right)$ such that $G_{i} \backslash x_{i}$ is connected, for $i=1, \ldots, k$. Hence by the induction hypothesis the connected components of $G \backslash x_{i}$ are $\mathrm{CIO}$ graphs. Since this is true for every $i=1, \ldots, k$ then $G_{1}, \ldots, G_{k}$ are $\mathrm{CI} \mathcal{O}$ graphs.

$(\Leftarrow)$ It is a consequence of Lemma 7.

Corollary 4 If $G$ is a CIO graph then every induced subgraph $H$ of $G$ is a $\mathrm{CIO}$ graph.

Proof Assume $H$ is not a $\mathrm{ClO}$ graph. By Proposition 5, $H$ has a connected component $H_{1}$ such that $H_{1}$ is not a $\mathrm{CIO}$ graph. Let $G_{1}$ be the connected component of $G$ such that $H_{1} \subset G_{1}$. If $G_{1} \neq H_{1}$, then by Lemma 6 there exists $x_{1} \in V\left(G_{1}\right) \backslash V\left(H_{1}\right)$ such that $G_{1}^{1}=G_{1} \backslash x_{1}$ is connected. Furthermore $H_{1}$ is an induced subgraph of $G_{1}^{1}$ thus, if $G_{1}^{1} \neq H_{1}$, then there exists $x_{2} \in V\left(G_{1}^{1}\right) \backslash V\left(H_{1}\right)$, such that $G_{1}^{2}=G_{1}^{1} \backslash x_{2}$ is a connected subgraph and $H_{1} \subset G_{1}^{2}$. We continue with this process until we obtain the connected subgraphs $G_{1}=G_{1}^{0}, G_{1}^{1}, \ldots, G_{1}^{k}=H_{1}$ such that $G_{1}^{i+1}=G_{1}^{i} \backslash x_{i+1}$. Since $H_{1}$ is not a CIO graph by Theorem $2 H_{1}=G_{1}^{k}, \ldots, G_{1}^{1}, G_{1}^{0}=G_{1}$ are not $\mathrm{CI} \mathcal{O}$ graphs. Therefore by Proposition 5, $G$ is not a CIO graph. This is a contradiction, therefore $H$ is a $\mathrm{CIO}$ graph.

Proposition $6 \mathrm{G}$ is a CIO graph if and only if every block $B$ of $G$ is a CIO graph.

Proof $(\Rightarrow)$ If $B$ is a block of $G$ then $B$ is an induced subgraph of $G$. Hence by Theorem $4, B$ is a $\mathrm{CIO}$ graph.

$(\Leftarrow)$ It is a consequence of Lemma 7 . 


\section{Chorded-theta graphs}

In this section we introduce chorded-theta graphs, the notion of transversal triangles in these graphs and theta-ring graphs. In Theorem 3 we describe the minimal chordedtheta graphs without transversal triangles. In this section we prove that theta-ring graphs are closed under 0,1,2-clique-sums and that chordal graphs are theta-ring graphs. With these results we obtain the main result of this section: theta-ring graphs can be constructed by clique-sums of complete graphs and/or cycles, or equivalently by 0,1 , 2-clique-sums of chordal graphs and/or cycles. Finally we prove that the minimal forbidden induced subgraphs for the characterization of theta-ring graphs are: prisms, pyramids, thetas and partial wheels that are chorded-thetas. This section is independent of the rest of the paper and its techniques are purely combinatorial.

Definition 4 A chorded-theta subgraph $T$ of $G$ is a subgraph induced by three paths $\mathcal{L}_{1}, \mathcal{L}_{2}, \mathcal{L}_{3}$ each between the non adjacent vertices $x$ and $y$ such that $V\left(\mathcal{L}_{i}\right) \cap$ $V\left(\mathcal{L}_{j}\right)=\{x, y\}$ for $1 \leq i<j \leq 3$. The edges that do not belong to any of the sets $E\left(\mathcal{L}_{1}\right), E\left(\mathcal{L}_{2}\right)$ and $E\left(\mathcal{L}_{3}\right)$ are called the chords of $T$. Furthermore if each chord of $T$ has its end vertices in different paths (of $\mathcal{L}_{1}, \mathcal{L}_{2}, \mathcal{L}_{3}$ ), then $T$ is called a simple chorded-theta subgraph.

To emphasize that $\mathcal{L}_{1}, \mathcal{L}_{2}$ and $\mathcal{L}_{3}$ are the three paths associated to $T$ we denote them by $\mathcal{L}_{i}(T)$ for $i=1,2,3$. On the other hand we denote by $\mathcal{L}_{i}^{o}$ the interior of $\mathcal{L}_{i}$, i.e., $\mathcal{L}_{i}^{o}=\mathcal{L}_{i} \backslash\{x, y\}$.

Definition 5 Let $T$ be a chorded-theta of $G$, with $\mathcal{L}_{1}(T)=\left(x, x_{1}, \ldots, x_{r_{1}}, y\right)$, $\mathcal{L}_{2}(T)=\left(x, y_{1}, \ldots, y_{r_{2}}, y\right)$ and $\mathcal{L}_{3}(T)=\left(x, z_{1}, \ldots, z_{r_{3}}, y\right)$. A transversal triangle $H$ of $T$ is a triangle in $G$ such that $V(H)=\left\{x_{i}, y_{j}, z_{k}\right\}$ for some $i, j, k$.

We define the following sets: $\Theta(G)=\{T \mid T$ is a chorded-theta of $G\}, \Theta_{\Delta}(G)=$ $\{T \in \Theta(G) \mid T$ has a transversal triangle $\}$ and $\Theta_{\Delta}^{c}(G)=\Theta(G) \backslash \Theta_{\Delta}(G)$. Moreover, a minimum chorded-theta subgraph $T$ without transversal triangles is a subgraph in $\Theta_{\Delta}^{c}(G)$ such that if $T^{\prime} \in \Theta_{\Delta}^{c}(G)$ then $|V(T)| \leq\left|V\left(T^{\prime}\right)\right|$.

Remark 1 If $T$ is a minimum chorded-theta subgraph without transversal triangles, then $T$ is a simple chorded-theta subgraph.

In the following two lemmas we assume that $T$ is a chorded-theta subgraph of $G$ with $\mathcal{L}_{1}(T)=\left(x, x_{1}, \ldots, x_{r_{1}}, y\right), \mathcal{L}_{2}(T)=\left(x, y_{1}, \ldots, y_{r_{2}}, y\right)$ and $\mathcal{L}_{3}(T)=$ $\left(x, z_{1}, \ldots, z_{r_{3}}, y\right)$, where $|V(T)|=r_{1}+r_{2}+r_{3}+2$.

Lemma 8 Let $T$ be a minimum chorded-theta subgraph without transversal triangles with $r_{1} \leq r_{2}$. If $\left\{x_{i}, y_{j}\right\}$ is a chord of $T$, then $(i, j) \in\left\{(1,1),\left(r_{1}, r_{2}\right)\right\}$ or $r_{1}=1$.

Proof By Remark 1, $T$ is a simple chorded-theta. Let $\left\{x_{i}, y_{j}\right\}$ be a chord of $T$. We can assume that $r_{1}>1$. Hence, $i<r_{1}$ or $1<i$.

Now, suppose $i<r_{1}$ and take the chorded-theta $T_{1}$ with $\mathcal{L}_{1}\left(T_{1}\right)=\left(x_{i}, \ldots, x_{r_{1}}, y\right)$, $\mathcal{L}_{2}\left(T_{1}\right)=\left(x_{i}, y_{j}, \ldots, y_{r_{2}}, y\right)$ and $\mathcal{L}_{3}\left(T_{1}\right)=\left(x_{i}, x_{i-1}, \ldots, x_{1}, x, z_{1}, \ldots, z_{r_{3}}, y\right)$. Since 
$T$ has no chords whose both end vertices are in $\mathcal{L}_{1}(T)$ and $T \in \Theta_{\Delta}^{c}(G)$, then $T_{1} \in \Theta_{\Delta}^{c}(G)$. Observe that $\left|V\left(T_{1}\right)\right|=|V(T)|-(j-1)$, so by the minimality of $|V(T)|$, we have $j=1$. We will prove that $i=1$, by contradiction we will assume $i>1$. Thus, we can take the chorded-theta $T_{2}$ with $\mathcal{L}_{1}\left(T_{2}\right)=\left(x, y_{1}, x_{i}\right), \mathcal{L}_{2}\left(T_{2}\right)=$ $\left(x, x_{1}, \ldots, x_{i}\right)$ and $\mathcal{L}_{3}\left(T_{2}\right)=\left(x, z_{1}, \ldots, z_{r_{3}}, y, x_{r_{1}}, \ldots, x_{i}\right)$. Again, since $T$ has no chords whose both end vertices are in $\mathcal{L}_{1}(T)$ and $T \in \Theta_{\Delta}^{c}(G)$, we have $T_{2} \in \Theta_{\Delta}^{c}(G)$. But $\left|V\left(T_{2}\right)\right|=|V(T)|-\left(r_{2}-1\right)$. Furthermore $1<r_{1} \leq r_{2}$ so $|V(T)|>\left|V\left(T_{2}\right)\right|$ which is a contradiction. Therefore $i=1$ and $(i, j)=(1,1)$.

Similarly, if $1<i$ we obtain $(i, j)=\left(r_{1}, r_{2}\right)$.

Lemma 9 Let $T$ be a minimum chorded-theta without transversal triangles such that $r_{1}=1$ and $r_{2} \leq r_{3}$. If there exist chords $e_{1}, e_{2}$ of $T$ with $e_{1}=\left\{x_{1}, z_{s_{1}}\right\}$ and $e_{2}=\left\{y_{j}, z_{s_{2}}\right\}$ then $\left\{x_{1}, y_{j}\right\} \notin E(G)$. Moreover $\left(s_{1}, j, s_{2}\right)=\left(r_{3}, 1,1\right)$ or $\left(s_{1}, j, s_{2}\right)=$ $\left(1, r_{2}, r_{3}\right)$.

Proof By Remark 1, $T$ is a simple chorded-theta.

Case (1) $s_{1} \leq s_{2}$. Suppose that $\left\{x_{1}, y_{j}\right\} \in E(G)$. Since $T \in \Theta_{\Delta}^{c}(G)$ then $\left\{x_{1}, z_{s_{2}}\right\} \notin$ $E(G)$ and $z_{s_{1}} \neq z_{s_{2}}$. Thus, we take a chorded-theta $T^{\prime}$ given by $\mathcal{L}_{1}\left(T^{\prime}\right)=$ $\left(z_{s_{2}}, y_{j}, x_{1}\right), \mathcal{L}_{2}\left(T^{\prime}\right)=\left(z_{s_{2}}, \ldots, z_{r_{3}}, y, x_{1}\right)$ and $\mathcal{L}_{3}\left(T^{\prime}\right)=\left(z_{s_{2}}, z_{s_{2}-1}, \ldots, z_{s_{1}}, x_{1}\right)$. Furthermore there is no chord whose both end vertices are in $\mathcal{L}_{3}(T)$, hence $T^{\prime} \in \Theta_{\Delta}^{c}(G)$. But $\left|V\left(T^{\prime}\right)\right|=|V(T)|-s_{1}-j+1<|V(T)|$ which is a contradiction. Therefore $\left\{x_{1}, y_{j}\right\} \notin E(G)$. Now, we consider the chorded-theta $T^{\prime \prime}$ given by $\mathcal{L}_{1}\left(T^{\prime \prime}\right)=\left(x_{1}, z_{s_{1}}, z_{s_{1}+1}, \ldots, z_{s_{2}}, y_{j}\right), \mathcal{L}_{2}\left(T^{\prime \prime}\right)=\left(x_{1}, x, y_{1}, \ldots, y_{j}\right)$ and $\mathcal{L}_{3}\left(T^{\prime \prime}\right)=$ $\left(x_{1}, y, y_{r_{2}}, y_{r_{2}-1}, \ldots, y_{j}\right)$. We have $T^{\prime \prime} \in \Theta_{\Delta}^{c}(G)$ because there is no chord with its both end vertices in $\mathcal{L}_{2}(T)$. But $\left|V\left(T^{\prime \prime}\right)\right|=|V(T)|-\left(s_{1}-1\right)-\left(r_{3}-s_{2}\right)$, then $s_{1}=1$ and $r_{3}=s_{2}$ because $T$ is minimum. If $j \neq r_{2}$ we can take a chorded-theta subgraph $T^{\prime \prime \prime}$, where $\mathcal{L}_{1}\left(T^{\prime \prime \prime}\right)=\left(y_{j}, \ldots, y_{r_{2}}, y\right), \mathcal{L}_{2}\left(T^{\prime \prime \prime}\right)=\left(y_{j}, \ldots, y_{1}, x, x_{1}, y\right)$ and $\mathcal{L}_{3}\left(T^{\prime \prime \prime}\right)=\left(y_{j}, z_{r_{3}}, y\right)$. Thus $T^{\prime \prime \prime} \in \Theta_{\Delta}^{c}(G)$ because $T$ is a simple chorded-theta together with the fact that $T \in \Theta_{\Delta}^{c}(G)$. But $\left|V\left(T^{\prime \prime \prime}\right)\right|=|V(T)|-\left(r_{3}-1\right)$ hence $r_{3}=1$. Since $r_{2} \leq r_{3}$ then $r_{2}=1=j$, which is a contradiction. Therefore $j=r_{2}$ and $\left(s_{1}, j, s_{2}\right)=\left(1, r_{2}, r_{3}\right)$.

Case (2) $s_{2} \leq s_{1}$. Similarly to the case (1). We obtain $\left\{x_{1}, y_{j}\right\} \notin E(G)$ and $\left(s_{1}, j, s_{2}\right)=\left(r_{3}, 1,1\right)$.

Definition 6 ( $\forall \theta \exists \Delta$-property) A graph $G$ is called a theta-ring graph if every chorded-theta of $G$ has a transversal triangle, in this case we say that $G$ has the $\forall \theta \exists \Delta$-property.

Remark $2 G$ is a theta-ring graph if and only if $\Theta_{\Delta}^{c}(G)=\emptyset$.

Theorem 3 Let $G$ be a graph. If $G$ is not a theta-ring graph then there exists a chorded-theta $T$ in $G$ where $\mathcal{L}_{1}(T)=\left(x, x_{1}, \ldots, x_{r_{1}}, y\right), \mathcal{L}_{2}(T)=\left(x, y_{1}, \ldots, y_{r_{2}}, y\right)$, $\mathcal{L}_{3}(T)=\left(x, z_{1}, \ldots, z_{r_{3}}, y\right)$, with $1 \leq r_{1} \leq r_{2} \leq r_{3}$ such that $T$ satisfies at least one of the following conditions:

(1) The chords of $T$ are contained in $\left\{\left\{y_{1}, z_{1}\right\},\left\{y_{r_{2}}, z_{r_{3}}\right\}\right\}$.

(2) $r_{1}=1$, and the chords of $T$ are contained in $\left\{\left\{x_{1}, z_{r_{3}}\right\},\left\{y_{1}, z_{1}\right\}\right\}$. 
(3) $r_{1}=1$, and the chords of $T$ have the form $\left\{x_{1}, z_{j}\right\}$ for some $j$.

(4) $r_{1}=1, r_{2}=1$ and the chords of $T$ have the form $\left\{x_{1}, y_{1}\right\}$ or $\left\{x_{1}, z_{j}\right\}$ for some $j$.

Proof Since $\Theta_{\Delta}^{c}(G) \neq \emptyset$. We can take a minimum chorded-theta $T$ without transversal triangles. Thus, $T$ is a simple chorded-theta. Let $\mathcal{L}_{1}(T)=\left(x, x_{1}, \ldots, x_{r_{1}}, y\right)$, $\mathcal{L}_{2}(T)=\left(x, y_{1}, \ldots, y_{r_{2}}, y\right)$ and $\mathcal{L}_{3}(T)=\left(x, z_{1}, \ldots, z_{r_{3}}, y\right)$ with $1 \leq r_{1} \leq r_{2} \leq r_{3}$. We can suppose that $\left|\mathcal{L}_{1}(T)\right| \leq\left|\mathcal{L}_{1}\left(T^{\prime}\right)\right|$ for all $T^{\prime} \in \Theta_{\Delta}^{c}(G)$ such that $\left|V\left(T^{\prime}\right)\right|$ is minimum. Also we assume that if $\left|\mathcal{L}_{1}(T)\right|=\left|\mathcal{L}_{1}\left(T^{\prime}\right)\right|$ then $\left|\mathcal{L}_{2}(T)\right| \leq\left|\mathcal{L}_{2}\left(T^{\prime}\right)\right|$.

Now, we suppose that $T$ does not satisfy property (1). Hence, there exists a chord of $T$ in one of the possible forms: $\left\{x_{l}, y_{j}\right\}$ or $\left\{x_{l}, z_{j}\right\}$ or $\left\{y_{i}, z_{k}\right\}$, where $(i, k) \notin\left\{(1,1),\left(r_{2}, r_{3}\right)\right\}$.

In the first case we suppose that there exists $e=\left\{y_{i}, z_{k}\right\}$, a chord of $T$, where $(i, k) \notin\left\{(1,1),\left(r_{2}, r_{3}\right)\right\}$. By Lemma 8 we have $r_{2}=1$ and $i=1$. Now, since $r_{1} \leq r_{2}$ then $r_{1}=1$. So, by Lemma 9, if there exists $\left\{x_{1}, z_{j}\right\} \in E(G)$ then $\left\{x_{1}, y_{1}\right\} \notin E(G)$ and $(j, i, k)=\left(r_{3}, 1,1\right)$ or $(j, i, k)=\left(1,1, r_{3}\right)$. Thus, we would be in case (2). Now, if $\left\{x_{1}, z_{j}\right\} \notin E(G)$ for every $j$ then the chords of $T$ have the form $\left\{x_{1}, y_{1}\right\}$ or $\left\{y_{1}, z_{j}\right\}$, and we are in case (4).

In the second case suppose there exists a chord $e$ of $T$ such that $e=\left\{x_{i}, y_{j}\right\}$ or $e=\left\{x_{i}, z_{j}\right\}$. We will prove that $r_{1}=1$. We argue by contradiction, assume that $r_{1}>1$ and $e=\left\{x_{i}, y_{j}\right\}$. By Lemma 8 , we have $(i, j) \in\left\{(1,1),\left(r_{1}, r_{2}\right)\right\}$. We can suppose that $(i, j)=\left(r_{1}, r_{2}\right)$ and take the chorded-theta graph $T_{1}$ where $\mathcal{L}_{1}\left(T_{1}\right)=\left(x, x_{1}, \ldots, x_{r_{1}}\right)$, $\mathcal{L}_{2}\left(T_{1}\right)=\left(x, y_{1}, \ldots, y_{r_{2}}, x_{r_{1}}\right)$ and $\mathcal{L}_{3}\left(T_{1}\right)=\left(x, z_{1}, \ldots, z_{r_{3}}, y, x_{r_{1}}\right)$. Since $T$ is a simple chorded-theta and $T \in \Theta_{\Delta}^{c}(G)$ then $T_{1} \in \Theta_{\Delta}^{c}(G)$. But $|V(T)|=\left|V\left(T_{1}\right)\right|$ and $\left|\mathcal{L}_{1}\left(T_{1}\right)\right|=\left|\mathcal{L}_{1}(T)\right|-1$, which is a contradiction. The same result is obtained if $e=\left\{x_{i}, z_{j}\right\}$. Hence, we obtain $r_{1}=1$.

Now, we will prove that if there exists a chord $e^{\prime}$ of $T$ such that $e^{\prime}=\left\{x_{1}, y_{u}\right\}$ then $T$ satisfies (4). First we will prove that $r_{2}=1$. Again we argue by contradiction, assume $r_{2}>1$. Hence, $u>1$ or $u<r_{2}$. Without loss of generality, take $u>1$ and consider the chorded-theta $T_{2}$ where $\mathcal{L}_{1}\left(T_{2}\right)=\left(x, x_{1}, y_{u}\right), \mathcal{L}_{2}\left(T_{2}\right)=\left(x, y_{1}, \ldots, y_{u}\right)$ and $\mathcal{L}_{3}\left(T_{2}\right)=\left(x, z_{1}, \ldots, z_{r_{3}}, y, y_{r_{2}}, \ldots, y_{u}\right)$. Since $T \in \Theta_{\Delta}^{c}(G)$ and there are no chords in $\mathcal{L}_{2}\left(T_{2}\right)$, then $T_{2} \in \Theta_{\Delta}^{c}(G)$. But $|V(T)|=\left|V\left(T_{2}\right)\right|,\left|\mathcal{L}_{1}(T)\right|=\left|\mathcal{L}_{1}\left(T_{2}\right)\right|$ and $\left|\mathcal{L}_{2}(T)\right|>$ $\left|\mathcal{L}_{2}\left(T_{2}\right)\right|$, which is a contradiction. Thus, $r_{1}=r_{2}=1$ and $e^{\prime}=\left\{x_{1}, y_{1}\right\}$. Suppose $T$ does not satisfy (4) then there must exist $e_{1}, e_{2} \in E(G)$ such that $e_{1}=\left\{x_{1}, z_{j_{1}}\right\}$ and $e_{2}=\left\{y_{1}, z_{j_{2}}\right\}$. But, by Lemma $9,\left\{x_{1}, y_{1}\right\} \notin E(G)$. This is a contradiction. Hence, $T$ satisfies (4).

Now, we can assume that $T$ does not have a chord of the form $e^{\prime}=\left\{x_{1}, y_{u}\right\}$ then $e=\left\{x_{1}, z_{j}\right\}$. Suppose $T$ does not satisfy (3), then there must exist $e_{3}=\left\{y_{i}, z_{k}\right\}$. By Lemma $9,(j, i, k) \in\left\{\left(r_{3}, 1,1\right),\left(1, r_{2}, r_{3}\right)\right\}$. Therefore $T$ satisfies (2).

Definition 7 A partial wheel $W$ is a graph where $V(W)=\left\{z, z_{1}, \ldots, z_{k}\right\}$ such that $C=\left(z_{1}, \ldots, z_{k}, z_{1}\right)$ is a cycle in $W$ and the edges of $W$ are the edges of $C$ and some edges between $z$ and vertices of $C$. $C$ is called the rim of $W$ and $z$ is called the center of $W$.

A prism is a graph consisting of two vertex-disjoint triangles $C_{1}=\left(x_{1}, x_{2}, x_{3}, x_{1}\right)$ and $C_{2}=\left(y_{1}, y_{2}, y_{3}, y_{1}\right)$, and three paths $L_{1}, L_{2}, L_{3}$ pairwise vertex-disjoint, such that each $L_{i}$ is a path between $x_{i}$ and $y_{i}$ for $i=1,2,3$ and the subgraph induced by $V\left(L_{i}\right) \cup V\left(L_{j}\right)$ is a cycle for $1 \leq i<j \leq 3$. 
A pyramid is a graph consisting of a vertex $w$, a triangle $C=\left(z_{1}, z_{2}, z_{3}, z_{1}\right)$, and three paths $P_{1}, P_{2}, P_{3}$, such that: $P_{i}$ is between $w$ and $z_{i}$ for $i=1,2,3 ; V\left(P_{i}\right) \cap$ $V\left(P_{j}\right)=\{w\}$ and the subgraph induced by $V\left(P_{i}\right) \cup V\left(P_{j}\right)$ is a cycle for $1 \leq i<$ $j \leq 3$; and at most one of the $P_{1}, P_{2}, P_{3}$ has only one edge.

A theta is a graph consisting of two non adjacent vertices $x$ and $y$, and three paths $P, Q, R$ with ends $x$ and $y$, such that the union of every two of $P, Q, R$ is an induced cycle.

Detecting prisms, pyramids and thetas has been widely studied, for example see $[2,10]$ and their references.

Definition 8 A partial wheel $T$ with $\operatorname{rim} C$ and center $z$ is called a $\theta$-partial wheel if $|V(C)| \geq 4$ and there exist two non adjacent vertices in $V(C) \cap N_{T}(z)$.

Remark 3 A theta is a chorded-theta without chords. Furthermore, if $W$ is a partial wheel then $W$ is a $\theta$-partial wheel if and only if $W$ is a chorded-theta.

Corollary $5 T$ is a minimal forbidden induced subgraph for the class of theta-ring graphs if and only if $T$ satisfies at least one of the following conditions:

(a) $T$ is a $\theta$-partial wheel.

(b) $T$ is a prism, pyramid or theta.

Proof First we will prove that if $T$ satisfies (a) or (b), then $T$ is a chorded-theta graph without transversal triangles and $T$ does not contain a proper chorded-theta subgraph.

If $T$ satisfies (a) with $\operatorname{rim} C=\left(z_{1}, z_{2}, \ldots, z_{r_{1}}, z_{1}\right)$ and center $z$, then there exist $z_{i}, z_{j} \in N_{T}(z)$ where $\left\{z_{i}, z_{j}\right\} \notin E(T)$. We can suppose that $i<j$, then $T$ is a chorded-theta graph with the paths $\mathcal{L}_{1}(T)=\left(z_{i}, z_{i+1}, \ldots, z_{j}\right), \mathcal{L}_{2}(T)=\left(z_{i}, z_{,}, z_{j}\right)$ and $\mathcal{L}_{3}(T)=\left(z_{i}, z_{i-1}, \ldots, z_{1}, z_{r_{1}}, z_{r_{1}-1}, \ldots, z_{j}\right)$. Furthermore, since $C$ does not contain chords then $T$ is a chorded-theta without transversal triangles. On the other hand if $T^{\prime}$ is a chorded-theta subgraph of $T$, then one terminal vertex is in $C$. Thus, one path of $T^{\prime}$ has the form $\left(z_{i_{1}}, z, z_{j_{1}}\right)$ with $\left\{z_{i_{1}}, z_{j_{1}}\right\} \notin E(T)$ and $i_{1}<j_{1}$. Hence, the others paths of $T^{\prime}$ are $\left(z_{i_{1}}, z_{i_{1}+1}, \ldots, z_{j_{1}}\right)$ and $\left(z_{i_{1}}, z_{i_{1}-1}, \ldots, z_{1}, z_{r_{1}}, z_{r_{1}-1}, \ldots, z_{j_{1}}\right)$. Therefore $V(T)=V\left(T^{\prime}\right)$ and $T=T^{\prime}$.

Now, if $T$ is a prism, then $T$ is a chorded-theta with $\mathcal{L}_{1}(T)=\left(x_{1}, x_{2}, L_{2}, y_{2}\right)$, $\mathcal{L}_{2}(T)=\left(x_{1}, L_{1}, y_{1}, y_{2}\right)$ and $\mathcal{L}_{3}(T)=\left(x_{1}, x_{3}, L_{3}, y_{3}, y_{2}\right)$. Furthermore $T$ has only two chords, then $T$ does not contain transversal triangles. On the other hand, if $T^{\prime}$ is a chorded-theta in $T$, then the terminal vertices are $x_{i}$ and $y_{j}$ for some $i, j \in\{1,2,3\}$. Since $x_{i}$ and $y_{j}$ have degree at least three in $T^{\prime}$, we have $V\left(T^{\prime}\right)=V(T)$ and $T^{\prime}=T$.

If $T$ is a pyramid, then we can suppose that $\left|E\left(P_{1}\right)\right| \geq 2$ and $T$ is a chorded-theta with $\mathcal{L}_{1}(T)=\left(z_{1}, P_{1}, w\right), \mathcal{L}_{2}(T)=\left(z_{1}, z_{2}, P_{2}, w\right)$ and $\mathcal{L}_{3}(T)=\left(z_{1}, z_{3}, P_{3}, w\right)$. Additionally if $T$ has only one chord then $T$ does not contain transversal triangles. Furthermore if $T^{\prime}$ is a chorded-theta in $T$ then the terminal vertices are $z_{i}$ and $w$ for some $i \in\{1,2,3\}$. Since $z_{i}$ and $w$ have degree at least 3 in $T^{\prime}$, we have $V\left(T^{\prime}\right)=V(T)$ and $T^{\prime}=T$.

Finally, if $T$ is a theta graph it is clear that $T$ is a minimal chorded-theta graph without transversal triangles. 
Now, to obtain the result it is only necessary to prove that if $T$ satisfies (1), (2), (3) or (4) of Theorem 3 then $T$ satisfies (a) or (b).

Case 1: $T$ satisfies (1). If $T$ has less than two chords and $T$ is neither a pyramid nor a theta, then $r_{3}=1$ and $r_{2}=r_{1}=1$. Thus, $T$ satisfies (a). Now, we can suppose that $T$ has exactly two chords. If $r_{2} \geq 2$ then $T$ is a prism. Furthermore if $r_{2}=1$ then $T$ satisfies (a).

Case 2: $T$ satisfies (2). If $T$ has less than two chords, and $T$ is neither a pyramid nor a theta then $r_{3}=1$ and $r_{2}=1$. Hence, $T$ satisfies (a). Now, we can suppose that $T$ has exactly two chords. If $r_{3} \geq 2$ then $T$ is a prism. Furthermore if $r_{3}=1$ then $r_{2}=r_{1}=1$ and $T$ satisfies (a).

If $T$ satisfies (3) or (4) then $T$ satisfies (a).

Definition 9 Let $G_{1}, G_{2}$ be graphs such that $G=G_{1} \cup G_{2}, K=G_{1} \cap G_{2}$. If $K$ is a complete graph with $|K|=k$, then $G$ is called the $k$-clique-sum (or clique-sum) of $G_{1}$ and $G_{2}$ in $K$.

Remark 4 Let $r_{i}$ be the number of connected components of $G_{i}$ for $i=1,2$. If $G$ is 0-clique-sum of $G_{1}$ and $G_{2}$ then the number of connected components of $G$ is $r_{1}+r_{2}$.

In our definition of clique-sum we do not allow to delete any of the edges of $K$.

Proposition 7 The clique-sum of theta-ring graphs is a theta-ring graph.

Proof Let $G_{1}$ and $G_{2}$ be theta-ring graphs. We assume that $G$ is a $k$-clique-sum of $G_{1}$ and $G_{2}$. We can suppose that $G=G_{1} \cup G_{2}$ (i.e., $G_{1}, G_{2} \subseteq G$ ) and $G_{1} \cap$ $G_{2}=K$ where $K$ is a $k$-complete subgraph of $G$. By contradiction suppose that $G$ is not a theta-ring graph. Hence, there exists a chorded-theta $H$ (of $G$ ) without transversal triangles, where $\mathcal{L}_{1}(H)=\left(x, x_{1}, \ldots, x_{r_{1}}, y\right), \mathcal{L}_{2}(H)=\left(x, y_{1}, \ldots, y_{r_{2}}, y\right)$ and $\mathcal{L}_{3}(H)=\left(x, z_{1}, \ldots, z_{r_{3}}, y\right)$. We can assume that $H$ is a simple chorded-theta. Since $\{x, y\} \notin E(G)$ then $\{x, y\} \cap\left(G_{1} \triangle G_{2}\right) \neq \emptyset$, where $G_{1} \triangle G_{2}$ is the symmetric difference. Thus, without loss of generality we can assume that $x \in V\left(G_{1}\right) \backslash V\left(G_{2}\right)$. If $y \in V\left(G_{2}\right) \backslash V\left(G_{1}\right)$ then $\mathcal{L}_{i}^{o}(H) \cap K \neq \emptyset$ for $i=1,2,3$. But $K$ is a complete graph therefore $H$ has a transversal triangle, which is not possible so $y \in V\left(G_{1}\right)$. On the other hand $G_{1}$ is a theta-ring graph, then $V(H)$ is not contained in $V\left(G_{1}\right)$. Hence, there exists $w \in V(H)$ such that $w \in V\left(G_{2}\right) \backslash V\left(G_{1}\right)$. We can suppose that $w \in \mathcal{L}_{1}^{o}(H)$, i.e., $w=x_{i}$ for some $1 \leq i \leq r_{1}$. Since $H$ is a simple chorded-theta, then $L_{1}=\left(x, x_{1}, \ldots, x_{i}=w\right)$ is a path between $G_{1} \backslash G_{2}$ and $G_{2} \backslash G_{1}$. Furthermore $K$ is a cutset and there exists $x_{i_{1}} \in V\left(L_{1}\right) \cap V(K)$ with $i_{1}<i$. On the other hand if $y \in V(K)$ then $\left\{y, x_{i_{1}}\right\} \in E(G)$ but this is not possible because $H$ is a simple chorded-theta. Thus, $y \in V\left(G_{1}\right) \backslash V\left(G_{2}\right)$ and $L_{2}=\left(w=x_{i}, x_{i+1}, \ldots, x_{r_{1}}, y\right)$ is a path between $G_{1} \backslash G_{2}$ and $G_{2} \backslash G_{1}$. Hence, there exists $x_{i_{2}} \in V\left(L_{2}\right) \cap V(K)$ with $i<i_{2}$. Since $x_{i_{1}}, x_{i_{2}} \in V(K)$ then $\left\{x_{i_{1}}, x_{i_{2}}\right\} \in E(G)$. This is a contradiction, because $H$ is a simple chorded-theta.

Lemma 10 Let $G$ be a 2-connected graph and let $H$ be an induced connected subgraph of $G$ with $|V(H)| \geq 2$. If $x \in V(G) \backslash V(H)$ then there exist paths $L_{1}$ and $L_{2}$ 
between $x$ and $H$ such that $V\left(L_{1}\right) \cap V\left(L_{2}\right)=\{x\}, V\left(L_{i}\right) \cap V(H)=\left\{a_{i}\right\}$ for $i=1,2$, and $a_{1} \neq a_{2}$.

Proof Let $y$ be a vertex in $H$. Since $G$ is 2-connected graph there exist paths $P_{1}=\left(x=x_{1}, x_{2}, \ldots, x_{s_{1}}=y\right)$ and $P_{2}=\left(x=y_{1}, y_{2}, \ldots, y_{s_{2}}=y\right)$ between $x$ and $y$, such that $V\left(P_{1}\right) \cap V\left(P_{2}\right)=\{x, y\}$. We define $j_{1}=\min \left\{k \mid x_{k} \in V(H)\right\}$ and $j_{2}=$ $\min \left\{k \mid y_{k} \in V(H)\right\}$. If $x_{j_{1}} \neq y_{j_{2}}$ we can take $L_{1}=\left(x=x_{1}, x_{2}, \ldots, x_{j_{1}}\right)$ and $L_{2}=$ $\left(x=y_{1}, y_{2}, \ldots, y_{j_{2}}\right)$. Hence, we can assume that $x_{j_{1}}=y_{j_{2}}$, but $V\left(P_{1}\right) \cap V\left(P_{2}\right)=$ $\{x, y\}$ therefore $x_{j_{1}}=y_{j_{2}}=y$. Since $|V(H)| \geq 2$ there must exist $a \in V(H) \backslash\{y\}$. Furthermore $G$ is 2-connected so $G \backslash y$ is connected, and there exists a path $P_{3}=$ $\left(x=z_{1}, \ldots, z_{s_{3}}=a\right)$ between $x$ and $a$ in $G \backslash y$. Now, we take $k_{1}=\max \left\{k \mid z_{k} \in\right.$ $\left.V\left(P_{1}\right) \cup V\left(P_{2}\right)\right\}, k_{2}=\min \left\{k \mid k \geq k_{1}\right.$ and $\left.z_{k} \in V(H)\right\}$. We can suppose that $z_{k_{1}} \in$ $V\left(P_{1}\right)$ and $z_{k_{1}}=x_{i_{1}}$. Finally we take $L_{1}=\left(x=x_{1}, \ldots, x_{i_{1}}=z_{k_{1}}, z_{k_{1}+1}, \ldots, z_{k_{2}}\right)$ and $L_{2}=P_{2}$.

Definition 10 Let $C$ be a cycle without chords. $C$ is called a hole if $C$ is not a triangle.

Lemma 11 Let $G$ be a theta-ring graph with a hole $C$. Let $x$ be a vertex of $G$ such that $x \notin V(C)$. If there exist paths $P_{1}$ and $P_{2}$ between $x$ and $C$ such that $V\left(P_{1}\right) \cap V\left(P_{2}\right)=\{x\}$ with $V\left(P_{i}\right) \cap V(C)=\left\{y_{i}\right\}$ for $i=1,2$, then $\left\{y_{1}, y_{2}\right\} \in E(C)$. Furthermore if $P$ is a path between $x$ and $C$ with $V(P) \cap V(C)=\{y\}$ then $y \in$ $\left\{y_{1}, y_{2}\right\}$.

Proof Set $C=\left(x_{1}, x_{2}, \ldots, x_{k}=x_{1}\right)$ where $x_{1}=y_{1}$ and $y_{2}=x_{s}$. By way of contradiction, suppose that $\left\{y_{1}, y_{2}\right\} \notin E(C)$. Thus, there exists a chorded-theta $H$ of $G$ with $\mathcal{L}_{1}(H)=\left(y_{1}, P_{1}, x, P_{2}, y_{2}\right), \mathcal{L}_{2}(H)=\left(y_{1}=x_{1}, x_{2}, \ldots, x_{s}=y_{2}\right)$, and $\mathcal{L}_{3}(H)=\left(y_{1}=x_{1}, x_{k}, x_{k-1}, \ldots, x_{s}=y_{2}\right)$ but $C$ does not have any chord, then $H$ has no transversal triangles. Hence, $\left\{y_{1}, y_{2}\right\} \in E(C)$. Now, if $P=\left(y=z_{1}, z_{2}, \ldots, z_{s_{2}}=\right.$ $x)$ is a path between $x$ and $C$ with $V(P) \cap V(C)=\{y\}$, we will prove that $y \in\left\{y_{1}, y_{2}\right\}$. By way of contradiction suppose that $y \notin\left\{y_{1}, y_{2}\right\}$. We take $k_{3}=$ $\min \left\{i \mid z_{i} \in V\left(P_{1}\right) \cup V\left(P_{2}\right)\right\}$, but $y \notin V\left(P_{1}\right) \cup V\left(P_{2}\right)$ so $k_{3}>1$. We can suppose that $z_{k_{3}} \in V\left(P_{1}\right)$. Set $P_{1}=\left(w_{1}=y_{1}, w_{2}, \ldots, w_{s_{1}}=x\right)$ and $z_{k_{3}}=w_{l}$. Thus, there exist paths $L_{1}=\left(y_{1}=w_{1}, w_{2}, \ldots, w_{l}=z_{k_{3}}\right), L_{2}=\left(y=z_{1}, z_{2}, \ldots, z_{k_{3}}\right)$, and $L_{3}=\left(y_{2}, P_{2}, x=w_{s_{1}}, w_{s_{1}-1}, \ldots, w_{l}=z_{k_{3}}\right)$ such that $V\left(L_{1}\right) \cap V(C)=\left\{y_{1}\right\}$, $V\left(L_{2}\right) \cap V(C)=\{y\}$ and $V\left(L_{3}\right) \cap V(C)=\left\{y_{2}\right\}$. Then, $\left\{y, y_{1}\right\},\left\{y, y_{2}\right\} \in E(C)$, but $\left\{y_{1}, y_{2}\right\} \in E(C)$ and $C$ is a hole, which is a contradiction. Therefore, $y \in\left\{y_{1}, y_{2}\right\}$.

Definition 11 A graph is chordal if each of its cycles of length at least 4 has a chord.

Proposition 8 ([14]) Chordal graphs are exactly the graphs that can be formed by clique-sums of complete graphs.

Theorem $4(\forall \theta \exists \Delta)$ Let $G$ be a graph. The following conditions are equivalent:

(i) $G$ is a theta-ring graph (i.e., $\left.\Theta_{\Delta}^{c}(G)=\emptyset\right)$.

(ii) $G$ can be constructed by 0,1,2-clique-sums of chordal graphs and/or cycles.

(iii) $G$ can be constructed by clique-sums of complete graphs and/or cycles. 
(iv) $G$ does not contain as induced subgraph any graph from the following families:

(a) $\theta$-partial wheels.

(b) Prisms, pyramids and thetas.

Proof (i) $\Leftrightarrow$ (iv) By Corollary 5 .

(iii) $\Rightarrow$ (i) Complete graphs and cycles are theta-ring graphs (since they do not contain chorded-thetas). The result follows from Proposition 7.

(ii) $\Rightarrow$ (iii) Suppose that $G$ can be constructed by 0,1,2-clique-sums of $H_{1}, \ldots, H_{s}$, where each $H_{i}$ is a chordal graph or cycle. If $s=1$, then $G$ is a cycle or chordal graph. Hence, $G$ satisfies (iii) by Proposition 8 . Now, we can suppose that $s>1$ and we take $G^{\prime}$ to be the induced subgraph obtained by $0,1,2$-clique-sum of $H_{1}, \ldots, H_{s-1}$ such that $G$ is the $j$-clique-sum of $G^{\prime}$ and $H_{S}$ where $j \in\{0,1,2\}$. Furthermore $\left|V\left(G^{\prime}\right) \cap V\left(H_{S}\right)\right| \leq j$. By induction hypothesis we see that $G^{\prime}$ can be constructed by clique-sums of $L_{1}, \ldots, L_{l_{1}}$, where each $L_{i}$ is a cycle or a complete subgraph of $G^{\prime}$. If $H_{s}$ is a cycle then we obtain the result. Hence, we can assume that $H_{S}$ is a chordal graph. Thus, by Proposition 8, we find that $H_{S}$ can be constructed by clique-sums of the complete subgraphs $K_{1}, K_{2}, \ldots, K_{l_{2}}$. Since $\left|V\left(G^{\prime}\right) \cap V\left(H_{s}\right)\right| \leq j$ we can suppose that $G^{\prime} \cap H_{S} \subset K_{u}$ for some $u \in\left\{1, \ldots, l_{2}\right\}$. Therefore $G$ can be constructed by clique-sums of $L_{1}, \ldots, L_{l_{1}}, K_{u}, K_{u+1}, \ldots, K_{l_{2}}, K_{u-1}, \ldots, K_{1}$.

(i) $\Rightarrow$ (ii) By induction on $|V(G)|$. We can suppose that $|V(G)|>1$. Let $B_{1}, \ldots, B_{s}$ be the blocks of $G$. If $s>1$ then by the induction hypothesis $B_{1}, \ldots, B_{s}$ satisfy (ii). But $G$ can be obtained as 0 or 1 -clique-sums of its blocks so $G$ satisfies (ii). Thus, we can assume that $s=1$ and $G=B_{1}$. We can suppose that $G$ is not a chordal graph. Thus, $G$ has a cycle $C$ without chords such that $C$ is not a triangle, i.e., $C$ is a hole. Since $G$ is a block we find that $G$ is 2-connected. Set $C=\left(x_{1}, \ldots, x_{k}, x_{k+1}=x_{1}\right)$ and we define

$$
C_{i}=\left\{\begin{array}{l|l}
x \in V(G \backslash C) & \begin{array}{l}
\text { there exist paths } L_{x}, L_{x}^{\prime} \text { between } x \text { and } C \text { such that } \\
V\left(L_{x}\right) \cap V\left(L_{x}^{\prime}\right)=\{x\}, V\left(L_{x}\right) \cap V(C)=\left\{x_{i}\right\} \\
\text { and } V\left(L_{x}^{\prime}\right) \cap V(C)=\left\{x_{i+1}\right\}
\end{array}
\end{array}\right\}
$$

for $i=1, \ldots, k$. We will prove that $C_{1}, \ldots, C_{k}$ is a partition of $V(G \backslash C)$. By Lemma 10 if $x \in V(G \backslash C)$ then there exist paths $L_{1}$ and $L_{2}$ between $x$ and $C$, such that $V\left(L_{1}\right) \cap V\left(L_{2}\right)=\{x\}$ and $V\left(L_{i}\right) \cap V(C)=\left\{y_{i}\right\}$ for $i=1,2$ with $y_{1} \neq y_{2}$. Hence, by Lemma 11, $\left\{y_{1}, y_{2}\right\} \in E(C)$. Thus, there exists $i \in\{1, \ldots, k\}$ such that $x_{i}=y_{1}$ and $y_{2}=x_{i-1}$ or $y_{2}=x_{i+1}$. So, $x \in V\left(C_{i}\right)$ or $x \in V\left(C_{i-1}\right)$. Therefore $V(G \backslash C)=\bigcup_{i=1}^{k} V\left(C_{i}\right)$. Now, we take $z \in V\left(C_{i}\right) \cap V\left(C_{j}\right)$ with $i \neq j$. By Lemma 11, we have $\left\{x_{i}, x_{i+1}\right\} \subset\left\{x_{j}, x_{j+1}\right\}$ and $\left\{x_{j}, x_{j+1}\right\} \subset\left\{x_{i}, x_{i+1}\right\}$. Then, $i=j$ which is a contradiction. Hence, $V\left(C_{i}\right) \cap V\left(C_{j}\right)=\emptyset$ and $V\left(C_{1}\right), \ldots, V\left(C_{r}\right)$ is a partition of $V(G \backslash C)$. Let $G_{i}$ be the subgraph induced by $V\left(C_{i}\right) \cup\left\{x_{i}, x_{i+1}\right\}$. By the definition of $C_{i}$ we have $V\left(L_{x}\right) \cup V\left(L_{x}^{\prime}\right) \subset V\left(G_{i}\right)$ for $x \in V\left(C_{i}\right)$, so $G_{i}$ is 2connected, and by the induction hypothesis $G_{i}$ can be obtained as 0,1,2-clique-sums of chordal graphs and cycles. We define $B_{1}$ as the 2-clique-sum of $C$ and $G_{1}$ at the edge $\left\{x_{1}, x_{2}\right\}$. Now, we will construct successively $B_{i+1}$ as the 2-clique-sum of $B_{i}$ and $G_{i+1}$ at the edge $\left\{x_{i+1}, x_{i+2}\right\}$ for $i=1, \ldots, k-1$. But $B_{k-1}=G$ therefore $G$ can be constructed as $0,1,2$-clique-sums of chordal graphs and/or cycles. 


\section{$5 \mathrm{CIO}$ graphs and theta-ring graphs}

In this section we prove that the $\mathrm{CIO}$ property is closed under $0,1,2$-clique-sums and that the chordal graphs are $\mathrm{CIO}$ graphs. In particular, we prove that if $D=G_{\mathcal{O}}$ is a tournament, then $P_{D}$ is a binomial complete intersection. Also we prove that prisms, pyramids, thetas and $\theta$-partial wheels (the forbidden induced subgraphs given in Corollary 5) are not $\mathrm{CIO}$ graphs. The main result in this section is the equivalence between $\mathrm{CIO}$ graphs and theta-ring graphs.

Definition 12 An oriented complete graph is called a tournament.

Proposition 9 ([11]) If $D_{n}=\left(K_{n}\right)_{\mathcal{O}_{n}}$ is a tournament, then $D_{n}$ has a Hamiltonian oriented path.

Lemma 12 Let $\mathcal{O}$ be an orientation of $G$ and $x$ a vertex of $G$ with $N_{G}(x)=$ $\left\{x_{1}, \ldots, x_{r_{1}}\right\}$ such that $P=\left(x_{1}, \ldots, x_{r_{1}}\right)$ is an oriented path in $D=G_{\mathcal{O}}$. Let $\mathcal{O}^{\prime}$ be the orientation of $G^{\prime}=G \backslash x$ induced by $\mathcal{O}$. If $C_{i}=\left(x, x_{i}, x_{i+1}, x\right)$, then $P_{D} \subseteq$ $\left(P_{D^{\prime}} \cup\left\{t_{C_{1}}, \ldots, t_{C_{r_{1}-1}}\right\}\right)$, where $D^{\prime}=G^{\prime} \mathcal{O}^{\prime}$.

Proof Since $P$ is an oriented path, we can assume that $\left(x_{j}, x_{j+1}\right) \in E(D)$ for $j=$ $1, \ldots, r_{1}-1$. For $1 \leq i<j \leq r_{1}$, we take the cycle $C_{i, j}=\left(x, x_{i}, P_{i, j}, x_{j}, x\right)$ where $P_{i, j}$ is the oriented subpath of $P$ that joins $x_{i}$ and $x_{j}$. First we will prove that $t_{C_{i, j}} \in$ $\left(t_{C_{1}}, \ldots, t_{C_{r_{1}-1}}\right)$ by induction on $j-i$. If $j-i=1$ it is clear. Using the induction hypothesis we have $t_{C_{i, j-1}} \in\left(t_{C_{1}}, \ldots, t_{C_{r_{1}-1}}\right)$. Thus, by Lemma 2 we have $t_{C_{i, j}} \in$ $\left(t_{C_{i, j-1}}, t_{C_{j-1}}\right)$. Therefore $t_{C_{i, j}} \in\left(t_{C_{1}}, \ldots, t_{C_{r_{1}-1}}\right)$.

Let $C$ be a cycle without chords of $D$. If $x \notin V(C)$ then $C \subset D^{\prime}$ and $t_{C} \in P_{D^{\prime}}$. Now, if $x \in V(C)$ then $C=\left(x, x_{j_{1}}, L, x_{j_{2}}, x\right)$ where $1 \leq j_{1}<j_{2} \leq r_{1}$ and $L$ is a path between $x_{j_{1}}$ and $x_{j_{2}}$ in $D$. Since $C$ is a cycle without chords, $V(L) \cap N_{G}(x)=$ $\left\{x_{j_{1}}, x_{j_{2}}\right\}$. On the other hand we take the cycle $C^{\prime}=\left(x_{j_{1}}, L, x_{j_{2}}, P_{j_{1}, j_{2}}, x_{j_{1}}\right)$, then $C^{\prime} \subseteq D^{\prime}$ and $t_{C^{\prime}} \in P_{D^{\prime}}$. By Lemma 2 we have $t_{C} \in\left(t_{C^{\prime}}, t_{C_{i, j}}\right)$. Furthermore $t_{C_{i, j}} \in\left(t_{C_{1}}, \ldots, t_{C_{r_{1}-1}}\right)$, then $t_{C} \in\left(P_{D^{\prime}}, t_{C_{1}}, \ldots, t_{C_{r_{1}-1}}\right)$. Therefore $P_{D} \subseteq\left(P_{D^{\prime}} \cup\right.$ $\left.\left\{t_{C_{1}}, \ldots, t_{C_{r_{1}-1}}\right\}\right)$, by Proposition 4 .

Definition 13 Let $G$ be a graph. A simplicial vertex $x$ is a vertex such that the induced subgraph obtained from $N_{G}(x)$ in $G$ is a complete graph.

Proposition 10 ([4]) If $G$ is a chordal graph, then $G$ has a simplicial vertex.

Theorem 5 If $G$ is a chordal graph then $G$ is a $C I O$ graph.

Proof By induction on $|V(G)|$. By Proposition 5 we can suppose that $G$ is a connected graph. Since $G$ is chordal there exists a vertex $x$ in $G$ such that $x$ is a simplicial vertex. Thus, if $K$ is the subgraph induced by $N_{G}(x)$, then $K$ is a complete graph and $G^{\prime}=G \backslash x$ is a connected subgraph of $G$. We can suppose that $N_{G}(x)=\left\{x_{1}, \ldots, x_{s}\right\}$. Let $\mathcal{O}$ be an orientation of $G$ and $D=G_{\mathcal{O}}$. By induction hypothesis $G^{\prime}$ is $\mathrm{CIO}$, hence if $\mathcal{O}^{\prime}$ is the orientation of $G^{\prime}$ induced by $\mathcal{O}$ and $D^{\prime}=G_{\mathcal{O}^{\prime}}^{\prime}$, then there exists the binomial generating set $\mathcal{G}^{\prime}$ of $P_{D^{\prime}}$ with $\left|\mathcal{G}^{\prime}\right|=(q-s)-(n-1)+1$. On the other hand, 
by Proposition 9, there exists a Hamiltonian oriented path $P$ of $K$. We can suppose that $P=\left(x_{1}, \ldots, x_{s}\right)$. We take the triangle $C_{i}=\left(x, x_{i}, x_{i+1}, x\right)$ for $i=1, \ldots, s-1$ and $\mathcal{G}=\mathcal{G}^{\prime} \cup\left\{t_{C_{1}}, \ldots, t_{C_{s-1}}\right\}$. Hence, by Lemma $12, \mathcal{G}$ is a generating set of $P_{D}$ and $|\mathcal{G}|=q-n+1$. Therefore $G$ is $\mathrm{CIO}$.

Corollary 6 If $D$ is a tournament, then $P_{D}$ is a binomial complete intersection.

Proof Since $D$ is a tournament then $D=K_{\mathcal{O}}$ where $K$ is a complete graph. Thus $K$ is a chordal graph and by Theorem $5 K$ is a CIO graph. Therefore $P_{D}$ is a binomial complete intersection.

Proposition 11 Let $G_{1}, G_{2}$ be two connected graphs and let $G$ be the 2-clique-sum of $G_{1}$ and $G_{2}$. If $G_{1}$ and $G_{2}$ are $C I \mathcal{O}$ graphs then $G$ is a $C I O$ graph.

Proof Let $\mathcal{O}$ be an orientation of the edges of $G$ and let $\mathcal{O}_{i}$ be the orientation of the edges of $G_{i}$ induced by $\mathcal{O}$, for $i=1$, 2. If $\left|V\left(G_{i}\right)\right|=n_{i}$ and $\left|E\left(G_{i}\right)\right|=q_{i}$, for $i=1,2$, then there exists a set $B_{i}$ with $q_{i}-n_{i}+1$ binomials such that $B_{i}$ generates $P_{D_{i}}$, where $D_{i}=\left(G_{i}\right)_{\mathcal{O}_{i}}$. Since $G$ is the 2-clique-sum of $G_{1}$ and $G_{2}$ then $|E(G)|=$ $q_{1}+q_{2}-1$ and $|V(G)|=n_{1}+n_{2}-2$. If $C$ is a cycle without chords of $D$ then $V(C) \subset V\left(D_{1}\right)$ or $V(C) \subset V\left(D_{2}\right)$. Hence, $t_{C} \in\left(B_{1} \cup B_{2}\right)$ and by Proposition 4, we see that $B_{1} \cup B_{2}$ is a generating set of $P_{G_{\mathcal{O}}}$. But

$$
\left|B_{1} \cup B_{2}\right|=\sum_{i=1}^{2}\left(q_{i}-n_{i}+1\right)=\left(q_{1}+q_{2}-1\right)-\left(n_{1}+n_{2}-2\right)+1=\operatorname{ht}\left(P_{G_{\mathcal{O}}}\right) .
$$

Therefore $G$ is $\mathrm{CIO}$.

If $L=\left(t_{i_{1}}, t_{i_{2}}, \ldots, t_{i_{s}}\right)$ is an oriented path in $D=G_{\mathcal{O}}$, we denote by $t_{L}$ the product of the edges of $L$, i.e., $t_{L}=t_{i_{1}} t_{i_{2}} \cdots t_{i_{s}}$.

Proposition 12 Prisms, pyramids, thetas and $\theta$-partial wheels are not $C I O$ graphs.

Proof Case (a). $T$ is a partial wheel where $V(T)=\left\{x_{1}, \ldots, x_{m}, x\right\}$ with center $x$, rim $C=\left(x_{1}, x_{2}, \ldots, x_{m}, x_{1}\right)$, and $N_{T}(x)=\left\{x_{j_{1}}, \ldots, x_{j_{k}}\right\}$.

First subcase, if $k \geq 4$ we take $\mathcal{T}=T_{\mathcal{O}}$ with the acyclic orientation $\mathcal{O}$ given into Fig. 1.

We have $\operatorname{ht}\left(P_{\mathcal{T}}\right)=k$. Let $\mathcal{G}=\left\{f_{1}, \ldots, f_{s}\right\}$ be a minimal binomial set of generators for $P_{\mathcal{T}}$. Since there are no oriented cycles in $\mathcal{T}$ and using Corollary 1 , then there is no binomial having as a monomial 1 . Since $g_{i} \in P_{\mathcal{T}}$, then there exists a binomial $f_{l_{i}} \in \mathcal{G}$ such that $f_{l_{i}}$ has as a monomial $t_{i}$ for $i=2, \ldots, k-2$ and $i=k$. Without loss of generality we can suppose that $l_{i}=i$ for $i=2, \ldots, k-2$ and $l_{k}=k-1$. Observe that if $C$ is a cycle of $T$ and $E(C) \cap E\left(L_{i}\right) \neq \emptyset$ then $E\left(L_{i}\right) \subset E(C)$. We will use this observation in the rest of the proof.

By the form of $g_{k+1}$ there exists a binomial $f_{j} \in \mathcal{G}$ with a monomial that divides $t_{L_{k-2}} t_{L_{k}}$. We can suppose that $f_{j}=t^{\alpha_{j}}-t^{\beta_{j}}$ and $t^{\alpha_{j}} \mid t_{L_{k-2}} t_{L_{k}}$. By Theorem 1 there exists a cycle $C_{1}$ such that the monomials of $t_{C_{1}}=t^{\alpha_{j}^{\prime}}-t^{\beta_{j}^{\prime}}$ divide the monomials 


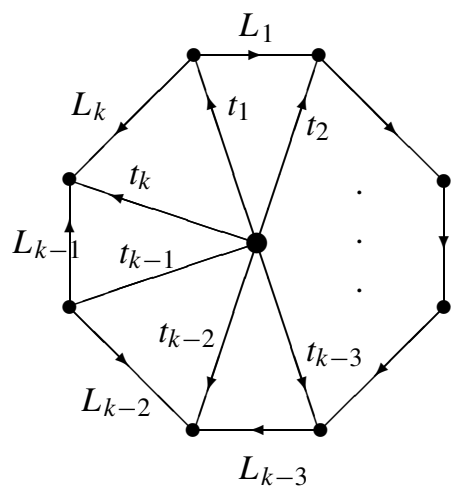

$$
\begin{aligned}
g_{1} & =t_{2}-t_{1} t_{L_{1}} \\
g_{2} & =t_{3}-t_{2} t_{L_{2}} \\
& \vdots \\
g_{i} & =t_{i+1}-t_{i} t_{L_{i}} \\
& \vdots \\
g_{k-3} & =t_{k-2}-t_{k-3} t_{L_{k-3}} \\
g_{k-2} & =t_{k-2}-t_{k-1} t_{L_{k-2}} \\
g_{k-1} & =t_{k}-t_{k-1} t_{L_{k-1}} \\
g_{k} & =t_{k}-t_{1} t_{L_{k}} \\
g_{k+1} & =t_{L_{k-2}} t_{L_{k}}-t_{L_{1}} \cdots t_{L_{k-3}} t_{L_{k-1}}
\end{aligned}
$$

Fig. 1 Acyclic orientation of the partial wheel $\mathcal{T}$ with $k \geq 4$, and the equations of its chordless cycles

of $f_{j}$. First we prove $E\left(L_{k}\right) \subset E\left(C_{1}\right)$. Suppose that it is not true, then $E\left(L_{k}\right) \cap$ $E\left(C_{1}\right)=\emptyset$. Hence, $E\left(L_{k-2}\right) \cap E\left(C_{1}\right) \neq \emptyset$ and $E\left(L_{k-2}\right) \subset E\left(C_{1}\right)$. Furthermore since $t^{\alpha_{j}^{\prime}} \mid t_{L_{k-2}} t_{L_{k}}$, then $t_{k-1} \notin E\left(C_{1}\right)$ and $E\left(L_{k-1}\right) \subset E\left(C_{1}\right)$. But $E\left(L_{k}\right) \cap E\left(C_{1}\right)=\emptyset$, then $t_{k} \in E\left(C_{1}\right)$ and $t_{k} \mid t^{\alpha_{j}^{\prime}}$. This is a contradiction because $t_{k}$ does not divide $t_{L_{k-2}} t_{L_{k}}$. Therefore $E\left(L_{k}\right) \subset E\left(C_{1}\right)$. Now, we prove that $x \notin E\left(C_{1}\right)$. Suppose $x \in E\left(C_{1}\right)$, we take the minimal $i_{1}=\min \left\{i \in\{1, \ldots, k\} \mid t_{i} \in E\left(C_{1}\right)\right\}$. Thus, $\bigcup_{i=1}^{i_{1}} E\left(L_{i}\right) \subset E\left(C_{1}\right)$ and $t_{i_{1}} \mid t^{\alpha_{j}^{\prime}}$. But this is not possible because $t_{i_{1}}$ does not divide $t_{L_{k-2}} t_{L_{k}}$. Hence, $x \notin$ $E\left(C_{1}\right)$ and $C_{1}=\mathcal{T} \backslash x$. Therefore $t_{C_{1}}=g_{k+1}$. Using Proposition 1 we have $f_{j}=$ $g_{k+1}$. Furthermore $f_{j} \neq f_{i}$ for $i=2, \ldots, k-1$, then we can suppose that $j=1$.

On the other hand by the form of $g_{k-1}$ there exists a binomial $f_{j_{1}} \in \mathcal{G}$ with a monomial that divides $t_{k-1} t_{L_{k-1}}$. By Theorem 1 there exists a cycle $C_{2}$ such that the monomials of $t_{C_{2}}=t^{\alpha_{j_{1}}^{\prime}}-t^{\beta_{j_{1}}^{\prime}}$ divide the monomials of $f_{j}$. We can suppose that $t^{\alpha_{j_{1}}^{\prime}} \mid t_{k-1} t_{L_{k-1}}$. Suppose $E\left(L_{k-1}\right) \cap E\left(C_{2}\right)=\emptyset$ then $t_{k-1} \in E\left(C_{2}\right)$ and $E\left(L_{k-2}\right) \subset$ $E\left(C_{2}\right)$. Thus, $t_{L_{k-2}} \mid t^{\alpha_{j_{1}}^{\prime}}$ which is not possible because $t_{L_{k-2}}$ does not divide $t_{k-1} t_{L_{k-1}}$. So, $E\left(L_{k-1}\right) \cap E\left(C_{2}\right) \neq \emptyset$ and $E\left(L_{k-1}\right) \subset E\left(C_{2}\right)$. Now, we prove that $t_{k-1} \in E\left(C_{2}\right)$. If $t_{k-1} \notin E\left(C_{2}\right)$ then $E\left(L_{k-2}\right) \subset E\left(C_{2}\right)$. Furthermore, $E\left(L_{k-3}\right) \subset E\left(C_{2}\right)$ or $t_{k-2} \in$ $E\left(C_{2}\right)$. Hence, $t_{L_{k-3}} \mid t^{\alpha_{j_{1}}^{\prime}}$ or $t_{k-2} \mid t^{\alpha_{j_{1}}^{\prime}}$. But this is not possible, then $t_{k-1} \in E\left(C_{2}\right)$. Now, $t_{k} \in E\left(C_{2}\right)$ or $E\left(L_{k}\right) \subset E\left(C_{2}\right)$. If $t_{k} \in E\left(C_{2}\right)$ then $C_{2}=\left(t_{k-1}, L_{k-1}, t_{k}\right)$ and $t_{C_{2}}=-g_{k-1}$. For the other case, if $E\left(L_{k}\right) \subset E\left(C_{2}\right)$ then $C_{2}=\left(t_{k-1}, L_{k-1}, L_{k}, t_{1}\right)$ and $t_{C_{2}}=t_{k-1} t_{L_{k-1}}-t_{1} t_{L_{k}}=g_{k}-g_{k-1}$. In both cases, by Proposition 1 we have $f_{j_{1}}=t_{C_{2}}$. Furthermore, by the form of $g_{k}$ there exists a binomial $f_{j_{2}} \in \mathcal{G}$ with a monomial that divides $t_{1} t_{L_{k}}$. In a similar way as in the last argument, we find that there exists a cycle $C_{3}$ such that $f_{j_{2}}=t_{C_{3}}$ where $C_{3}=\left(t_{k}, L_{k}, t_{1}\right)$ or $C_{3}=$ $\left(t_{k-1}, L_{k-1}, L_{k}, t_{1}\right)$. So, $f_{j_{1}}, f_{j_{2}} \in\left\{t_{k}-t_{k-1} t_{L_{k-1}}, t_{k}-t_{1} t_{L_{k}}, t_{k-1} t_{L_{k-1}}-t_{1} t_{L_{k}}\right\}$. If $\left\{f_{1}, \ldots, f_{k-1}\right\} \cap\left\{f_{j_{1}}, f_{j_{2}}\right\} \neq \emptyset$ then $f_{j_{1}}=f_{k-1}$ or $f_{j_{2}}=f_{k-1}$ and in this case $f_{j_{1}} \neq f_{j_{2}}$. Therefore $k \leq\left|\left\{f_{1}, \ldots, f_{k-1}, f_{j_{1}}, f_{j_{2}}\right\}\right|$.

By the form of $g_{k-2}$ there exists a binomial $f_{j_{3}}$ with a monomial that divides $t_{k-1} t_{L_{k-2}}$. Thus, there exists a cycle $C_{4}$ such that the monomials of $t_{C_{4}}=t^{\alpha_{j_{3}}^{\prime}}-t^{\beta_{j_{3}}^{\prime}}$ divide the monomials of $f_{j_{3}}$. We can suppose that $t^{\alpha_{j_{3}}^{\prime}} \mid t_{k-1} t_{L_{k-2}}$. Since $t^{\alpha_{j_{3}}^{\prime}} \neq 1$ 


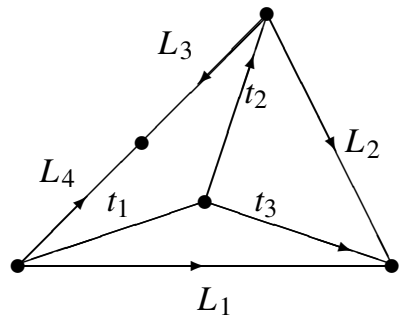

$$
\begin{aligned}
& g_{1}=t_{3}-t_{1} t_{L_{1}} \\
& g_{2}=t_{3}-t_{2} t_{L_{2}} \\
& g_{3}=t_{1} t_{L_{4}}-t_{2} t_{L_{3}} \\
& g_{4}=t_{L_{1}} t_{L_{3}}-t_{L_{2}} t_{L_{4}}
\end{aligned}
$$

Fig. 2 Acyclic orientation of the partial wheel $\mathcal{T}$ with $k=3$, and the equations of its chordless cycles

and $t_{L_{k-1}}$ does not divide $t^{\alpha_{j_{3}}^{\prime}}$ then $V\left(L_{k-2}\right) \subset V\left(C_{4}\right)$. Furthermore, since $t_{L_{k}}$ and $t_{k}$ do not divide $t^{\alpha^{\prime}}$ then $t_{k-1} \in E\left(C_{4}\right)$. Hence, if $t_{k-2} \in E\left(C_{4}\right)$ then $C_{4}=$ $\left(t_{k-2}, L_{k-2}, t_{k-1}\right)$ and $t_{C_{4}}=t_{k-1} t_{L_{k-2}}-t_{k-2}$. In the other case, if $t_{k-2} \notin E\left(C_{4}\right)$ then $t_{C_{4}}=t_{k-1} t_{L_{k-2}}-t^{\beta_{j_{3}}^{\prime}}$. Thus, $t^{\alpha_{j_{3}}^{\prime}}=t_{k-1} t_{L_{k-2}}$ and by Proposition 1 we have $f_{j_{3}}=t_{C_{4}}$. Furthermore, if $t_{k-2} \notin E\left(C_{4}\right)$ then $k+1 \leq\left|\left\{f_{1}, \ldots, f_{k-1}, f_{j_{1}}, f_{j_{2}}, f_{j_{3}}\right\}\right|$ and $P_{\mathcal{T}}$ is not a binomial complete intersection. So, we can assume that $t_{k-2} \in$ $E\left(C_{4}\right)$ and $f_{j_{3}}=t_{k-1} t_{L_{k-2}}-t_{k-2}$. Moreover, if $f_{j_{3}} \neq-f_{k-2}$ then $k+1 \leq$ $\left|\left\{f_{1}, \ldots, f_{k-1}, f_{j_{1}}, f_{j_{2}}, f_{j_{3}}\right\}\right|$ and $P_{\mathcal{T}}$ is not a binomial complete intersection. Hence, we can assume $-f_{k-2}=f_{j_{3}}=t_{k-1} t_{L_{k-2}}-t_{k-2}$ and it is the unique binomial in $\mathcal{G}$ which has a monomial that divides either $t_{k-2}$ or $t_{k-1} t_{L_{k-2}}$. Then,

$$
\begin{aligned}
t_{k-2}-t_{k-3} t_{L_{k-3}} & =\sum_{i=1}^{s} g_{i} f_{i}=g_{k-2}\left(t_{k-1} t_{L_{k-2}}-t_{k-2}\right)+\sum_{i \neq k-2} g_{i} f_{i} \\
& =\left(-1+g_{k-2}^{\prime}\right)\left(t_{k-1} t_{L_{k-2}}-t_{k-2}\right)+\sum_{i \neq k-2} g_{i} f_{i} .
\end{aligned}
$$

Here $\operatorname{deg}\left(g_{k-2}^{\prime}\right)>0$ or $g_{k-2}^{\prime}=0$. Using the last equation we obtain

$$
t_{k-1} t_{L_{k-2}}-t_{k-3} t_{L_{k-3}}=g_{k-2}^{\prime}\left(t_{k-1} t_{L_{k-2}}-t_{k-2}\right)+\sum_{i \neq k-2} g_{i} f_{i}
$$

So, there exists a $f_{i}$ with $i \neq k-2$ where one of its monomials divides $t_{k-1} t_{L_{k-2}}$. But this is not possible.

Second subcase, if $k=3$ we take $\mathcal{T}=T_{\mathcal{O}}$ with the acyclic orientation $\mathcal{O}$ given in Fig. 2.

We have $\operatorname{ht}\left(P_{\mathcal{T}}\right)=3$. Let $\mathcal{G}=\left\{f_{1}, \ldots, f_{S}\right\}$ be a minimum binomial set of generators of $P_{\mathcal{T}}$. For every $m \in A=\left\{t_{3}, t_{1} t_{L_{1}}, t_{2} t_{L_{2}}, t_{1} t_{L_{4}}, t_{2} t_{L_{3}}, t_{L_{1}} t_{L_{3}}, t_{L_{2}} t_{L_{4}}\right\}$ there exists a binomial $f \in \mathcal{G}$ such that $f$ has a monomial $t^{\alpha}$ and $t^{\alpha} \mid m$. By Theorem 1 there exists a cycle $C$ such that $t_{C}=t^{\alpha^{\prime}}-t^{\beta^{\prime}}$ and $t^{\alpha^{\prime}} \mid t^{\alpha}$. But there are no cycles whose binomials have either $t_{L_{i}}$ or $t_{j}$ as a monomial, for $i=1, \ldots, 4$ and $j=1,2$. Hence, $t^{\alpha^{\prime}}=t^{\alpha}=m$ and by Proposition $1 t_{C}=f$. Then, for every $m \in A$ there exists $f \in \mathcal{G}$ such that $m$ is a monomial of $f$. But $|A| / 2 \leq|\mathcal{G}|$ and $|A|=7$ then $4 \leq|\mathcal{G}|$. Therefore $P_{\mathcal{T}}$ is not a binomial complete intersection. 


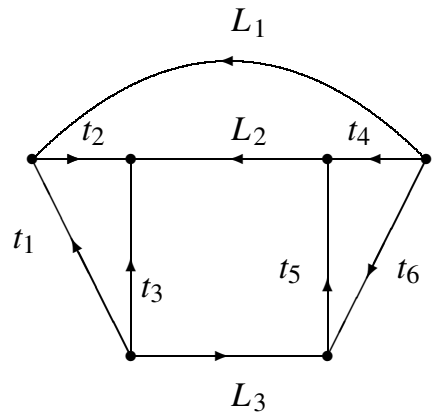

$$
\begin{aligned}
& g_{1}=t_{4}-t_{5} t_{6} \\
& g_{2}=t_{3}-t_{1} t_{2} \\
& g_{3}=t_{3}-t_{L_{3}} t_{5} t_{L_{2}} \\
& g_{4}=t_{1} t_{6}-t_{L_{1}} t_{L_{3}} \\
& g_{5}=t_{4} t_{L_{2}}-t_{2} t_{L_{1}}
\end{aligned}
$$

Fig. 3 Acyclic orientation of the prism $\mathcal{T}$, and the equations of its cycles without chords

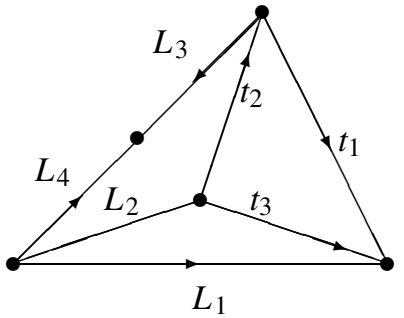

$$
\begin{aligned}
& g_{1}=t_{3}-t_{L_{1}} t_{L_{2}} \\
& g_{2}=t_{3}-t_{1} t_{2} \\
& g_{3}=t_{L_{2}} t_{L_{4}}-t_{2} t_{L_{3}} \\
& g_{4}=t_{L_{1}} t_{L_{3}}-t_{1} t_{L_{4}}
\end{aligned}
$$

Fig. 4 Acyclic orientation of the pyramid $\mathcal{T}$, and the equations of its cycles without chords

Case (b-1). If $T$ is a prism, we take $\mathcal{T}=T_{\mathcal{O}}$ with the acyclic orientation $\mathcal{O}$ given in Fig. 3.

We have $\operatorname{ht}\left(P_{\mathcal{T}}\right)=4$. Let $\mathcal{G}=\left\{f_{1}, \ldots, f_{s}\right\}$ be a minimal binomial set of generators of $P_{\mathcal{T}}$. We take $A=\left\{t_{5} t_{6}, t_{1} t_{2}, t_{2} t_{L_{1}}, t_{L_{3}} t_{5} t_{L_{2}}, t_{1} t_{6}, t_{L_{1}} t_{L_{3}}, t_{3}, t_{4}\right\}$. By the same argument as for partial wheels with $k=3$, we see that for every $m \in A$ there exist $f \in \mathcal{G}$ and a cycle $C$ of $\mathcal{T}$ such that $m$ is a monomial of $f$ and $f=t_{C}$. Let $f_{j_{1}}$, $f_{j_{2}}, f_{j_{3}}$ be binomials of $\mathcal{G}$ that have as a monomial $t_{3}, t_{1} t_{2}$ and $t_{L_{3}} t_{5} t_{L_{2}}$, respectively. Hence, if $f_{j_{1}}=t_{C_{1}}, f_{j_{2}}=t_{C_{2}}$ and $f_{j_{3}}=t_{C_{3}}$, then

$$
C_{1}, C_{2}, C_{3} \in\left\{\left(t_{3}, t_{1}, t_{2}\right),\left(t_{1}, t_{2}, L_{2}, t_{5}, L_{3}\right),\left(t_{3}, L_{3}, t_{5}, L_{2}\right)\right\} .
$$

Thus, if $B=\left\{f_{j_{1}}, f_{j_{2}}, f_{j_{3}}\right\}$ then $|B| \geq 2$. Since if $f_{j_{1}}=f_{j_{3}}$ then $C_{2}=C_{3}=$ $\left(t_{1}, t_{2}, L_{2}, t_{5}, L_{3}\right)$ and $f_{j_{1}} \neq f_{j_{2}}$. Furthermore for every monomial in $A^{\prime}=A \backslash$ $\left\{t_{3}, t_{1} t_{2}, t_{L_{3}} t_{5} t_{L_{2}}\right\}$ there exists a binomial in $\mathcal{G} \backslash B$. But $|\mathcal{G} \backslash B| \geq\left|A^{\prime}\right| / 2$ and $\left|A^{\prime}\right|=5$ then $|\mathcal{G} \backslash B| \geq 3$ and $|\mathcal{G}| \geq 5$. Therefore $P_{\mathcal{T}}$ is not a binomial complete intersection.

Case (b-2). If $T$ is a pyramid, we take $\mathcal{T}=T_{\mathcal{O}}$ with the acyclic orientation $\mathcal{O}$ given in Fig. 4.

This case is similar to the case when $T$ is a partial wheel with $k=3$, only change $t_{1}$ by the path $L_{2}$. Therefore $P_{\mathcal{T}}$ is not a binomial complete intersection.

Case (b-3) If $T$ is a theta, we take $\mathcal{T}=T_{\mathcal{O}}$ with the acyclic orientation $\mathcal{O}$ given in Fig. 5.

We have $\operatorname{ht}\left(P_{\mathcal{T}}\right)=2$. Let $\mathcal{G}=\left\{f_{1}, \ldots, f_{S}\right\}$ be a minimum binomial set of generators of $P_{\mathcal{T}}$. We take $A=\left\{t_{L_{1}} t_{L_{4}}, t_{L_{2}} t_{L_{3}}, t_{L_{3}} t_{L_{6}}, t_{L_{4}} t_{L_{5}}, t_{L_{1}} t_{L_{6}}, t_{L_{2}} t_{L_{5}}\right\}$. By the same 


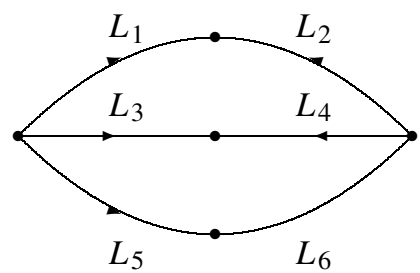

$$
\begin{aligned}
& g_{1}=t_{L_{1}} t_{L_{4}}-t_{L_{2}} t_{L_{3}} \\
& g_{2}=t_{L_{3}} t_{L_{6}}-t_{L_{4}} t_{L_{5}} \\
& g_{3}=t_{L_{1}} t_{L_{6}}-t_{L_{2}} t_{L_{5}}
\end{aligned}
$$

Fig. 5 Acyclic orientation of the theta $\mathcal{T}$, and the equations of its cycles without chords

argument as for partial wheels with $k=3$, we find that for every $m \in A$ there exist $f \in \mathcal{G}$ and a cycle $C$ of $\mathcal{T}$ such that $m$ is a monomial of $f$ and $f=t_{C}$. Hence, $|\mathcal{G}| \geq|A| / 2$ but $|A|=6$ then $|\mathcal{G}| \geq 3$. Therefore $P_{\mathcal{T}}$ is not a binomial complete intersection.

Theorem 6 Let $G$ be a graph. $G$ is a CIO graph if and only if $G$ is a theta-ring graph.

Proof $(\Rightarrow)$ If $G$ is not a theta-ring graph, then by Theorem $4, G$ has an induced subgraph $H$ that is either a prism, pyramid, theta or $\theta$-partial wheel. Thus, by Proposition $12, H$ is not a $\mathrm{CIO}$ graph. Hence, by Corollary $4, G$ is not a $\mathrm{CIO}$ graph which is a contradiction. Therefore $G$ is a theta-ring graph.

$(\Leftarrow)$ By Theorem $4 G$ can be constructed by 0,1 , 2-clique-sums of chordal graphs and/or cycles. By Proposition 5 chordal graphs and cycles are $\mathrm{CIO}$ graphs. Therefore by Proposition 5, Proposition 6 and Proposition 11, $G$ is a $\mathrm{CIO}$ graph.

Definition 14 ([5]) A graph $G$ is a ring graph if each block of $G$ which is not a bridge or a vertex can be constructed successively by 2-clique-sums of cycles.

Corollary 7 ([6]) If $G$ is a ring graph, then $G$ is a $C I O$ graph. The converse holds if $G$ is bipartite.

Proof First we observe that ring graphs can be obtained by 0,1,2-clique-sums of vertices, edges and cycles. Hence, by Theorem $4, G$ is a theta-ring graph and by Theorem $6, G$ is a $\mathrm{CIO}$ graph.

Now, if $G$ is bipartite and $G$ is a $\mathrm{CIO}$ graph, then by Theorem 4 and Theorem 6 , $G$ can be constructed by $0,1,2$-clique-sums of chordal graphs and/or cycles. Since $G$ is bipartite, if $H$ is a chordal induced subgraph of $G$, then $H$ is a forest. Hence, $G$ can be constructed by $0,1,2$-clique-sums of cycles and/or edges. Therefore, $G$ is a ring graph.

Acknowledgements The authors thank ABACUS, CONACyT grant EDOMEX-2011-C01-165873.

\section{References}

1. Atiyah, M.F., Macdonald, I.G.: Introduction to Commutative Algebra. Addison-Wesley, Reading (1969) 
2. Chudnovsky, M., Kapadia, R.: Detecting a theta or prism. SIAM J. Discrete Math. 22(3), 1164-1186 (2008)

3. Diestel, R.: Graph Theory, 2nd edn. Graduate Texts in Mathematics, vol. 173. Springer, New York (2000)

4. Dirac, G.A.: On rigid circuit graphs. Abh. Math. Semin. Univ. Hamb. 25(1-2), 71-76 (1961)

5. Gitler, I., Reyes Villarreal, R.H.: Ring graphs and toric ideals. Electron. Notes Discrete Math. C 28, 393-400 (2007)

6. Gitler, I., Reyes, E., Villarreal, R.H.: Ring graphs and complete intersection toric ideals. Discrete Math. 310, 430-441 (2010)

7. Gitler, I., Villarreal, R.H.: Graphs, Rings and Polyhedra. Textos Nivel Avanzado. Aportaciones Matemáticas, vol. 35. Sociedad Matemática Mexicana, Mexico (2011)

8. Katsabekis, A.: Arithmetical rank of toric ideals associated to graphs. Proc. Am. Math. Soc. 138(9), 3111-3123 (2010)

9. Katzman, M.: Bipartite graphs whose edge algebras are complete intersections. J. Algebra 220, 519_ 530 (1999)

10. Lévêque, B., Lin, D.Y., Maffray, F., Trotignon, N.: Detecting induced subgraphs. Discrete Appl. Math. 157, 3540-3551 (2009)

11. Rédei, L.: Ein kombinatorischer Satz. Acta Litt. Szeged 7, 39-43 (1934)

12. Reyes, E.: Complete intersection toric ideals of oriented graphs. Morfismos 9(2), 71-82 (2005)

13. Reyes, E., Tatakis, C., Thoma, A.: Minimal generators of toric ideals of graphs. Adv. Appl. Math. 48, 64-72 (2012)

14. Seymour, P.D., Weaver, R.W.: A generalization of chordal graphs. J. Graph Theory 8(2), 241-251 (1984)

15. Simis, A.: On the Jacobian module associated to a graph. Proc. Am. Math. Soc. 126, 989-997 (1998)

16. Tatakis, C., Thoma, A.: On the universal Gröbner bases of toric ideals of graphs. J. Comb. Theory, Ser. A 118, 1540-1548 (2011)

17. Villarreal, R.H.: Rees algebras of edge ideals. Commun. Algebra 23, 3513-3524 (1995)

18. Villarreal, R.H.: Monomial Algebras. Monographs and Textbooks in Pure and Applied Mathematics, vol. 238. Marcel Dekker, New York (2001) 